\title{
A whole genome duplication drives the genome evolution of Phytophthora betacei, a closely related species to Phytophthora infestans
}

David A. Ayala-Usma ${ }^{1,2,3}$, Martha Cárdenas ${ }^{3}$, Romain Guyot ${ }^{4,5}$, Maryam Chaib De Mares ${ }^{1,2}$, Adriana Bernal ${ }^{6}$, Alejandro Reyes Muñoz ${ }^{1,2,7^{*}}$ and Silvia Restrepo ${ }^{3^{*}}$

\begin{abstract}
Background: Pathogens of the genus Phytophthora are the etiological agents of many devastating diseases in several high-value crops and forestry species such as potato, tomato, cocoa, and oak, among many others. Phytophthora betacei is a recently described species that causes late blight almost exclusively in tree tomatoes, and it is closely related to Phytophthora infestans that causes the disease in potato crops and other Solanaceae. This study reports the assembly and annotation of the genomes of $P$. betacei P8084, the first of its species, and $P$. infestans RC1-10, a Colombian strain from the EC-1 lineage, using long-read SMRT sequencing technology.

Results: Our results show that $P$. betacei has the largest sequenced genome size of the Phytophthora genus so far with $270 \mathrm{Mb}$. A moderate transposable element invasion and a whole genome duplication likely explain its genome size expansion when compared to $P$. infestans, whereas $P$. infestans RC1-10 has expanded its genome under the activity of transposable elements. The high diversity and abundance (in terms of copy number) of classified and unclassified transposable elements in $P$. infestans RC1-10 relative to $P$. betacei bears testimony of the power of long-read technologies to discover novel repetitive elements in the genomes of organisms. Our data also provides support for the phylogenetic placement of $P$. betacei as a standalone species and as a sister group of $P$. infestans. Finally, we found no evidence to support the idea that the genome of $P$. betacei P8084 follows the same gene-dense/gense-sparse architecture proposed for $P$. infestans and other filamentous plant pathogens.

Conclusions: This study provides the first genome-wide picture of $P$. betacei and expands the genomic resources available for $P$. infestans. This is a contribution towards the understanding of the genome biology and evolutionary history of Phytophthora species belonging to the subclade 1c.
\end{abstract}

\footnotetext{
* Correspondence: a.reyes@uniandes.edu.co; srestrep@uniandes.edu.co ${ }^{1}$ Research Group in Computational Biology and Microbial Ecology, Department of Biological Sciences, Universidad de los Andes, Bogotá, Colombia

${ }^{3}$ Laboratory of Mycology and Plant Pathology (LAMFU), Department of

Chemical and Food Engineering, Universidad de Los Andes, Bogotá, Colombia

Full list of author information is available at the end of the article
}

(c) The Author(s). 2021 Open Access This article is licensed under a Creative Commons Attribution 4.0 International License, which permits use, sharing, adaptation, distribution and reproduction in any medium or format, as long as you give appropriate credit to the original author(s) and the source, provide a link to the Creative Commons licence, and indicate if changes were made. The images or other third party material in this article are included in the article's Creative Commons licence, unless indicated otherwise in a credit line to the material. If material is not included in the article's Creative Commons licence and your intended use is not permitted by statutory regulation or exceeds the permitted use, you will need to obtain permission directly from the copyright holder. To view a copy of this licence, visit http://creativecommons.org/licenses/by/4.0/ The Creative Commons Public Domain Dedication waiver (http://creativecommons.org/publicdomain/zero/1.0/) applies to the data made available in this article, unless otherwise stated in a credit line to the data. 


\section{Background}

Pathogens of the genus Phytophthora are the etiological agents of many devastating diseases in a wide range of plant hosts, that include several high-value crops and forestry species such as potato, tomato, cocoa, and oak among many others [1, 2]. A notorious species of the genus is $P$. infestans, the causal agent of late blight of potato and tomato. This disease caused the Irish famine, a phenomenon that led to the exodus and death of millions of people in Ireland during the 19th Century [3, 4]. In the last decade, the southern region of Colombia has been affected by an increased incidence of late blight leading to devastating economic losses in tree tomato (Solanum betaceum) crops. Mideros et al. [5] described the novel species $P$. betacei as the causal agent of the mentioned late blight of the tree tomato, instead of the sympatrically occurring $P$. infestans that causes the disease in potato crops.

Phytophthora betacei species was erected and distinguished from $P$. infestans and its closely related species $P$. andina using morphological, physiological, molecular, and host-specificity evidence in isolates obtained from crops with late blight [5]. The molecular evidence, based on simple sequence repeat (SSR) and genotyping-bysequencing (GBS) markers, allowed the classification of isolates previously classified as the EC-3 clonal lineage of Phytophthora infestans sensu lato (using RFLP data [6]) into the putative $P$. betacei species. Furthermore, the data from 22788 SNPs revealed a clear genetic structure among the three evaluated species, except for two individuals (likely misclassified) of $P$. andina clustering within the EC-3 lineage along with $P$. betacei individuals. This suggested that $P$. infestans, $P$. andina, and $P$. betacei are genetic differentiated groups with negligible gene flow between them [5].

Although GBS data provides valuable genetic evidence to support speciation, there is no public genome assembly or sequencing data for $P$. betacei that allows for further inquiry. Also, the lack of public genome assemblies of $P$. infestans from Colombia and the Americas limits the research on genomic-scale evolution and regional genetic diversity of the late blight pathogens. Whole genome sequences are needed to perform structural and comparative genomics analyses aiming to explore the species diversification dynamics in these Phytophthora species.

The nuclear genome of Phytophthora species is highly plastic; the term "two-speed genomes" has been coined [7] for this genus, due to their evolutionary dynamics, in a manner similar to other filamentous plant pathogens. The term means that the core orthologous housekeeping genes of the individual are distributed in gene-dense regions under purifying selection, whereas its pathogenicity and virulence-related genes are distributed along gene-sparse regions with high content of transposable elements and repeats under positive selection $[7,8]$. Some of these virulence-related genes, named effectors, are used by the pathogen to promote infection by modulating the immune response of the host. This is carried out by their interaction with apoplastic or cytoplasmic molecular targets and they may determine the pathogenicity in a susceptible host [9]. Effectors such as those from the RxLR and Crinkler (CRN) families translocate inside host cells $[10,11]$ and constitute two of the most studied virulence-related genes in the genus Phytophthora and tend to be located in gene-sparse regions $[8,12]$. It has been observed that extensive gene duplication, gene loss, and genomic rearrangements have occurred in the genomes of $P$. infestans, $P$. sojae, and $P$. ramorum, particularly in gene-sparse regions where effector genes are located, and evidence points to bursts of lineage-specific transposon activation as the cause of such phenomena $[8,13]$.

Repetitive content in Phytophthora species, particularly in the gene-sparse regions described above, represents an important fraction of the total genomic content, accounting for up to $74 \%$ of the sequenced bases in P. infestans T30-4 [12]. This repetitive content also represents a big challenge for accurate assembly with short-read sequencing technologies [14], as those used up to date, to reconstruct the representative assemblies of all members of the genus $[2,12,15]$. The emergence in recent years of longread sequencing technologies such as PacBio SMRT or Oxford Nanopore sequencing has unveiled a whole new panorama for Phytophthora genomics. Indeed, by yielding single-molecule reads of thousands of base pairs in length per run in any of these platforms, there is a reduced chance of mis-assembling highly repetitive regions, as long as the repeat is shorter than the read length. An additional advantage of these platforms is preventing subrepresentation of GC-rich and low-complexity genomic regions since they do not rely on PCR amplification of the source genomic material [16]. In summary, long read technologies pose the potential of yielding more accurate assemblies for the complex genomic architectures of the Phytophthora genus compared with previous short-read technologies.

Beside transposable elements, it has been reported that independent whole genome duplications (WGD) have shaped two closely related Phytophthora pathogens affecting Cacao: P. palmivora and P. megakarya $[17,18]$. As a consequence of WGD and invasion of transposable elements, the genomes of $P$. palmivora and $P$. megakarya are particularly large (135 and $222 \mathrm{Mb}$ ). Mideros et al. [5] estimated the nuclear DNA content of $P$. betacei isolate P8084 to be almost twice as large (1.13 pg) as that of the reference strain $P$. infestans T30-4 (0.67 pg), thus raising the question of whether a transposon-driven genomic expansion or a WGD occurred independently in this lineage. 
In this study, we perform genome assembly and annotation of $P$. betacei P8084 and a Colombian strain of $P$. infestans (RC1-10) using long-read SMRT sequencing technologies. The genome assembly size of $P$. betace $i$ is about $270 \mathrm{Mb}$, the largest assembled genome in the Phytophthora genus. A moderate transposable element invasion and a WGD likely explain its genome size expansion when compared to $P$. infestans. This is the first approach towards the understanding of the genomic expansion and evolutionary history of this novel Phytophthora species.

\section{Results}

\section{Genome sequencing, polishing, and haploid representation}

The complete sequencing dataset for $P$. betacei P8084 was composed by $\sim 17.5 \mathrm{~Gb}$ of uncorrected PacBio Sequel subreads combined with $\sim 22.7 \mathrm{~Gb}$ of Illumina paired-end (PE) reads generated in a previous study [5]. For $P$. infestans RC1-10 only PacBio Sequel sequencing was carried out and the yield of the uncorrected subreads was $\sim 9.2 \mathrm{~Gb}$.

A k-mer coverage frequency analysis of the Illumina data obtained for P. betacei P8084 showed a very likely scenario for a highly-heterozygous diploid organism (heterozygosity rate $=3.51 \%$ ) (Supplementary Fig. 1), after which we performed the assembly using only longreads and assuming both organisms to be diploid. To account for the high heterozygosity and the fact that this assembly is unphased, we performed haplotig curation. Afterwards, we used a two-step polishing approach to correct for indels and misassigned bases in the assemblies.

The final assembly statistics are shown in Table 1 . We also included the publicly available reference genomes of the genus, namely $P$. nicotianae, $P$. sojae, and $P$. ramorum RefSeq assemblies, as comparison. The RefSeq genome of $P$. infestans T30-4 served as the gold standard. Phytophthora betacei P8084 has a genome size of $270 \mathrm{Mb}$, the largest of all the evaluated assemblies, distributed among 802 contigs. It was $42 \mathrm{Mb}$ and $70 \mathrm{Mb}$ larger than $P$. infestans T30-4 and $P$. infestans RC1-10 genomes, respectively, and almost $200 \mathrm{Mb}$ (3.4X) larger than other Phytophthora genomes, constituting a more significant assembly challenge. The majority of the sequenced bases $(98.3 \%)$ were found in contigs larger than $50 \mathrm{~kb}$. The contig number of P. betacei P8084 is one order of magnitude greater than $P$. sojae, within the same order of magnitude as P. nicotianae, and one order of magnitude lower than the other assemblies. The contiguity of $P$. betacei assembly, as measured by the N50, is $\sim 100 \mathrm{~kb}$, making it one order of magnitude less contiguous than $P$. infestans T30-4 and P. sojae, but equivalent to the rest of the genomes. Phytophthora sojae assembly metrics show the largest N50 value, the smallest contig number, and largest percentage of bases sequenced in contigs $>=50 \mathrm{~kb}$, however it should be considered that is approximately $1 / 3$ of the genome size. $P$. betacei was placed second for two of such statistics with a significantly larger genome.

The $P$. infestans RC1-10 assembly has a total size of $203 \mathrm{Mb}$ contained in 2902 contigs, being smaller than the gold standard T30-4 genome which contains $228.5 \mathrm{Mb}$. It was also more fragmented with only $76.9 \%$ of the sequenced bases present in contigs larger than $50 \mathrm{~kb}$ compared to $89.9 \%$ in T30-4. Both P. infestans assemblies have a higher contig count and lower values for bases present in large contigs, but the RefSeq assembly showed a much higher N50 than RC1-10. An important advantage of using long-read assembly is that despite the quality differences among the assemblies generated in this study, P. betacei P8084 and P. infestans RC1-10 genomes have $0 \% \mathrm{~N}$ 's in the assembly, compared to $16.81 \%$ in the P. infestans T30-4 RefSeq genome.

Table 1 Assembly statistics of Phytophthora betacei P8084 and Phytophthora infestans RC1-10 compared to Phytophthora RefSeq genomes

\begin{tabular}{|c|c|c|c|c|c|c|}
\hline Assembly & $\begin{array}{l}\text { P. betacei } \\
\text { P8084 }\end{array}$ & $\begin{array}{l}\text { P. infestans } \\
\text { (RC1-10) }\end{array}$ & $\begin{array}{l}\text { P. infestans RefSeq } \\
\text { (T30-4) }\end{array}$ & $\begin{array}{l}\text { P. sojae RefSeq (V3 } \\
\text { P6497) }\end{array}$ & $\begin{array}{l}\text { P. nicotianae } \\
\text { (P1569) }\end{array}$ & $\begin{array}{l}\text { P. ramorum RefSeq } \\
\text { (Pr102) }\end{array}$ \\
\hline Contigs/Scaffolds & 802 & 2902 & 4921 & 82 & 5010 & 2576 \\
\hline Total length (Mb) & 270.89 & 203.29 & 228.54 & 82.60 & 55.10 & 66.65 \\
\hline $\begin{array}{l}\text { Genome in contigs > = } \\
50 \mathrm{~Kb}(\%)\end{array}$ & 98.29 & 76.90 & 89.88 & 99.12 & 27.90 & 72.63 \\
\hline Largest contig (Mb) & 4.15 & 0.88 & 6.93 & 13.39 & 0.30 & 1.24 \\
\hline GC (\%) & 51.00 & 51.00 & 51.00 & 55.00 & 49.60 & 54.00 \\
\hline N50 (Kb) & 737.97 & 120.01 & 1588.62 & 7609.24 & 27.20 & 308.04 \\
\hline L50 & 103.00 & 413.00 & 38.00 & 4.00 & 549.00 & 63.00 \\
\hline N's in the assembly (\%) & 0.00 & 0.00 & 16.81 & 3.96 & 0.00 & 18.35 \\
\hline
\end{tabular}

See Methods section for the accession numbers of the RefSeq genomes in Genbank database

[This table should appear in the same page than the Results section named Genome sequencing, polishing, and haploid representation] 
We also evaluated the semantic content of the genomes with BUSCO to have an estimate of gene recovery for each assembly. Of all the Phytophthora genomes evaluated for presence of single-copy orthologs in a Stramenopila-Alveolata dataset, P. betacei P8084 showed the fewest number of missing genes, followed by $P$. nicotianae. Phytophthora betacei P8084 also showed the highest number of duplicated genes of the evaluated panel. Phytophthora infestans RC1-10 showed a missing gene content greater than that of the other genomes but has comparable metrics of fragmented and duplicated genes relative to all the other species (Fig. 1). Together, these data suggest that P. betacei P8084 is one of the most semantically complete genomes of the genus, and it might have extensive duplication of core orthologs compared to all other compared assemblies. Also, it is a hint that $P$. infestans RC1-10 has an acceptable assembly quality comparable to public RefSeq genomes.

\section{Gene prediction and functional annotation}

MAKER 2 gene prediction and annotation pipeline was executed for both $P$. betacei P8084 and P. infestans RC110 with the transcriptome and proteome of $P$. infestans T30-4 as evidence to support gene prediction. This pipeline was also run for P. infestans T30-4 in a similar way for comparison purposes. The prediction yielded a total of 23457 genes for $P$. betacei P8084, 15893 genes for $P$. infestans RC1-10 and 17475 genes for $P$. infestans T304 with 14929 (63.64\%), 9680 (60.91\%) and 10506 (60.12\%) of the transcripts, respectively, having an assigned function as evidenced by domain identification via InterProScan or BLAST searches against UniRef90 database (Table 2). Uncharacterized proteins without assigned function represented between 36 and $39 \%$ of the total predicted genes for all three genomes, although novel proteins that did not match any previously reported uncharacterized proteins comprised only $~ 2 \%$ of the total gene content of each genome. In total, P. betacei P8084 showed a slightly larger proportion of genes relative to the sequenced genome size compared to both $P$. infestans assemblies (Table 2).

A search for carbohydrate-active enzymes (CAZymes) revealed 760 proteins in P. betacei P8084, 478 proteins in $P$. infestans RC1-10, and 492 proteins in P. infestans T30-4. The majority of the recovered proteins belonged to Glycoside Hydrolases ( $45 \%$ of all CAZymes in the three genomes) and Glycosyltransferases ( $\sim 25 \%$ of all CAZymes in the three genomes). The proportions of each category were highly similar across assemblies (Table 2). Based on these results, a trophic classification was carried out to quickly assess whether differences exist in CAZymes profiles among the three assemblies. All three assemblies were classified as monomertrophs (pathogens that consume mainly simple sugars) according to their highest relative centroid distance (RCD) score for each of the three nomenclatures proposed in CATAStrophy [19] (Supplementary Table 1). This trophic phenotype is compatible with the classic biotroph category of plant pathogens.

We then counted the number of virulence-associated genes in the genome annotation files from the three assemblies. This was carried out as a naive approach to estimate differences in genes relevant for plant-pathogen interaction. In general terms, the count of genes in the eight evaluated categories was similar for all three genomes. Our preliminary analysis showed that Phytophthora betacei P8084 generally

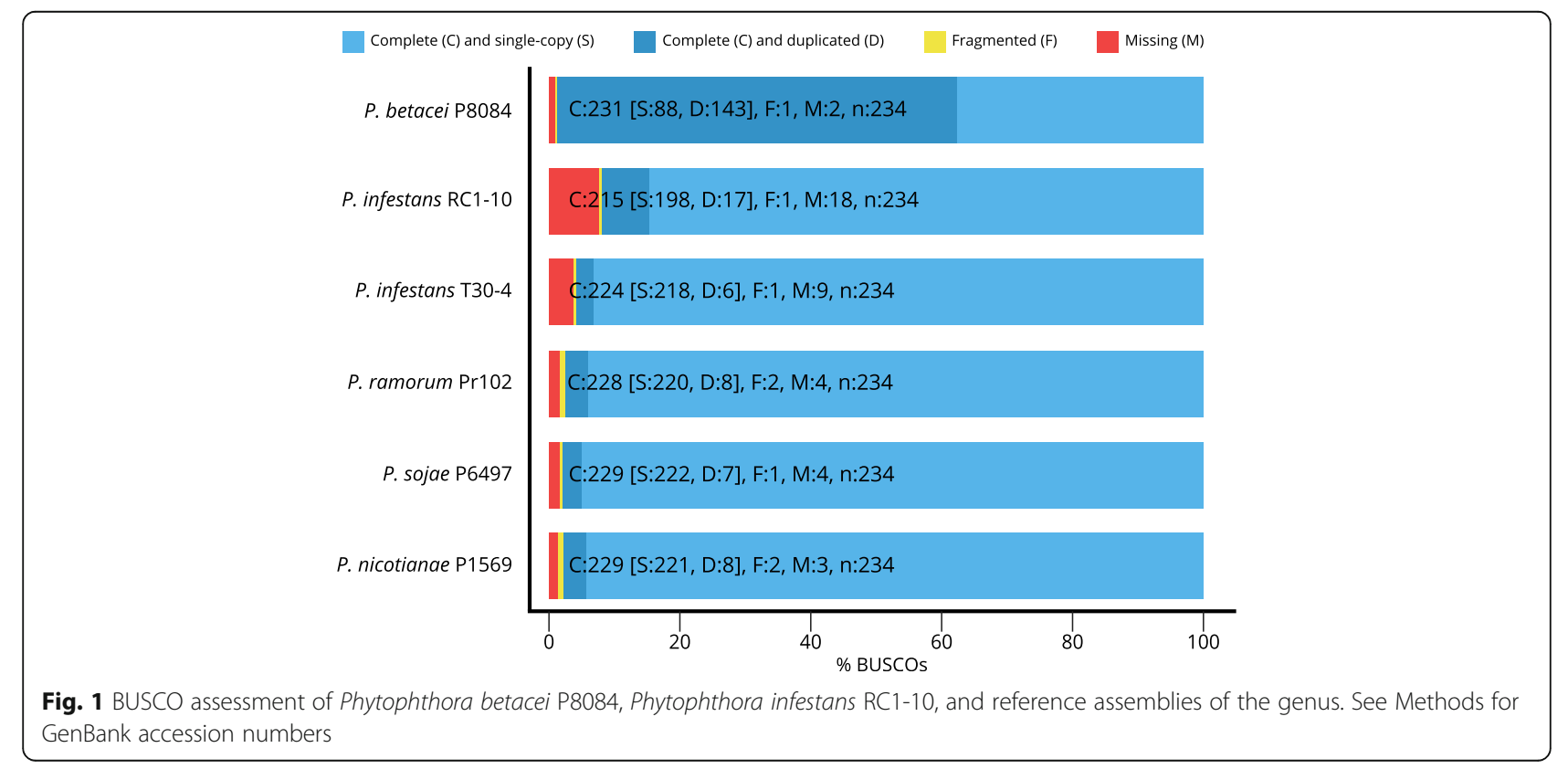


Table 2 Gene annotation statistics from MAKER2 and CAZymes for Phytophthora betacei P8084 and P. infestans assemblies

\begin{tabular}{llll}
\hline Assembly & P. betacei P8084 & P. infestans RC1-10 & P. infestans T30-4 \\
\hline MAKER 2 annotation & & & 15893 \\
Total genes & 23457 & 8771 & 9545 \\
Genes annotated via InterPro, GO, Pfam, and SUPERFAMILY terms. & 13659 & 909 & 961 \\
Genes annotated uniquely via BLAST against UniRef90 & 1270 & 5789 & 6553 \\
Genes matching uncharacterized proteins in UniRef90 & 8001 & 424 & 416 \\
Genes without assigned functional annotation & 527 & 22155935 & 25324938 \\
Cummulative gene coverage (bp) & 34360829 & $11.08 \%$ \\
Percentage of the genome covered by genes & $12.68 \%$ & $10.89 \%$ & 217 \\
Proteins with CAZymes functional annotation & & 220 & 124 \\
Glycoside Hydrolase Family & 327 & 115 & 36 \\
Glycosyltransferase Family & 187 & 30 & 32 \\
Polysaccharide Lyase Family & 54 & 38 & 51 \\
Carbohydrate Esterases & 67 & 47 & 32 \\
Carbohydrate-Binding Module Family & 78 & 34 & \\
Auxiliary Activities & 48 & & \\
\hline
\end{tabular}

Genes without assigned functional annotation: Those without matches to either Uniref90 or InterPro databases. Genes annotated via InterPro, GO, Pfam, and SUPERFAMILY terms: Counted as the number of gene elements in the GFF file with entries in the Dbxref field. Genes annotated uniquely via BLAST against UniRef90: Genes with functional terms derived from BLAST matches but without domain-related information from InterPro. Genes matching uncharacterized proteins in UniRef90: Genes with BLAST match to proteins labeled as uncharacterized that do not have associated functional domains. Cummulative gene coverage (bp): Cummulative number of bases from gene elements in the GFF file

[This table should appear in the same page than the Results section named Gene prediction and functional annotation]

has a larger number of genes per category relative to both $P$. infestans strains (Supplementary Table 2). Phytophthora betacei P8084 had almost twice the number of RxLR effectors (201) relative to $P$. infestans $\mathrm{RC} 1-10$ and $P$. infestans T30-4 (107). Crinkler (CRN) effectors were the second most abundant virulence-related genes in $P$. betacei P8084, however $P$. infestans T30-4 had the highest number of genes for the category broadly surpassing the other two assemblies. Surprisingly, P. infestans RC1-10 had the lowest number of CRNs of the three assemblies, breaking the similarity pattern with $P$. infestans T30-4 observed for the other categories (Supplementary Table 2).

\section{Orthologous genes analyses}

In order to provide a comparative context to the gene annotation of the novel assemblies, an analysis of orthologous families was carried out among the predicted proteomes of our genomes and those from the assemblies of $P$. nicotianae, $P$. sojae, $P$. ramorum, and $P$. infestans T30-4 since those are some of the most complete genomes of the genus so far. The 122388 proteins in the dataset were grouped in 20738 clusters, of which 18345 were orthologous clusters that contained proteins from at least two species, and 2393 were single-copy gene clusters (Fig. 2 A). The core genome of the analyzed genomes is composed of 5881 clusters of orthologs containing 42198 proteins from all species. This is the largest overlap partition observed in the analysis (Fig. 2B-C). Interestingly, proteins from $P$. betacei P8084 had a larger relative abundance $(21.99 \%)$ in the core genome relative to those belonging to any of the other genomes $(\sim 15 \%$ each). A total of 3806 (64.7\%) of the core genome clusters had associated Gene Ontology (GO) terms. These terms are related to housekeeping functions such as cellular metabolic process (GO:0044237), nitrogen compound metabolic process (GO:0006807), RNA metabolic process (GO:0016070), and response to stimulus (GO:0050896) among many others (Supplementary Table 3 ).

The 2137 clusters that were exclusive to $P$. betacei P8084 and P. infestans RC1-10, the genomes generated with long-read sequencing, comprised the second most numerous overlap partition (Fig. 2B-C). The vast majority of the clusters of this partition (2003 clusters, $93.7 \%$ of the total) did not have associated GO terms or any annotation at all. Those clusters that had associated GO terms, had functions similar to those from the core genome list, with housekeeping functions such as metabolism of carbohydrates and other macromolecules being the most abundant (Supplementary Tables 3 and 4). A total of $72.1 \%$ of the genes belonging to this partition can be found inside regions annotated as transposons, including but not limited to the transposases, (1415 out of 2389 proteins from P. betacei P8084 and 2020 from 2375 proteins from $P$. infestans $\mathrm{RC} 1-10)$ whereas the remaining $27.9 \%$ were genes found outside TE- or SSRrich areas (Supplementary Fig. 2). 


\begin{tabular}{|c|c|c|c|}
\hline |Species & | Proteins & |Orthologous clusters| & Singletons \\
\hline Phytophthora betacei P8084 & 23.457 & 13.061 & 4.687 \\
\hline Phytophthora infestans RC1-10 & 15.893 & 11.861 & 2.468 \\
\hline Phytophthora infestans T30-4 & 17.785 & 16.339 & 2.540 \\
\hline Phytophthora nicotianae & 23.159 & 13.899 & 6.509 \\
\hline Phytophthora ramorum & 15.605 & 10.822 & 1.499 \\
\hline Phytophthora sojae & 26.489 & 14.218 & 5.063 \\
\hline
\end{tabular}

The evaluated protein set from all species forms 20.738 different clusters: 18.345 orthologous clusters with representatives in at least two species and 2.393 single-copy gene clusters.

B
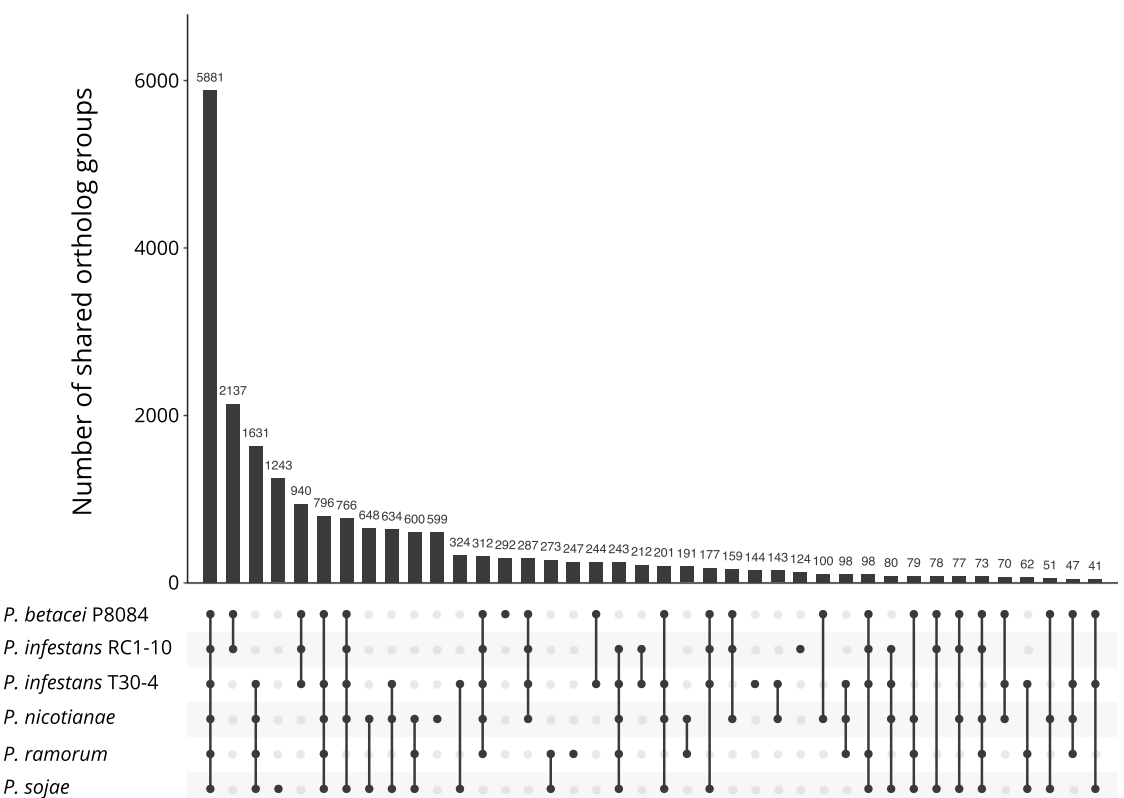

C

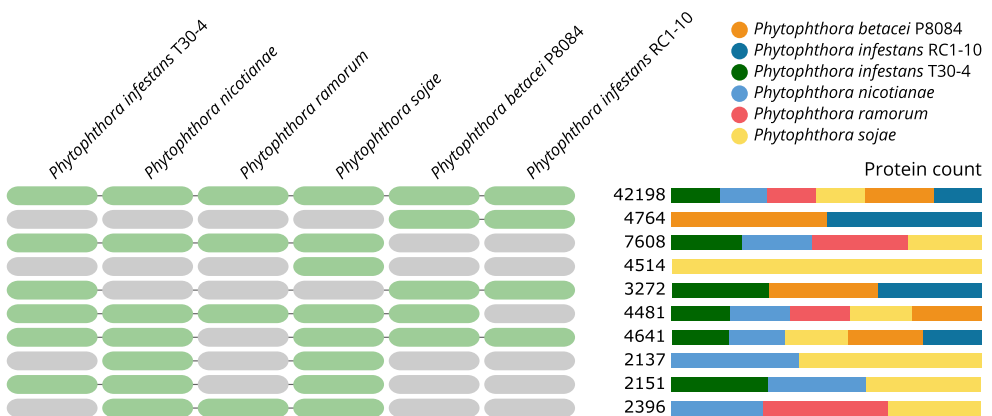

Fig. 2 Ortholog analysis for Phytophthora betacei P8084, Phytophthora infestans RC1-10, and reference assemblies of the genus. (A) Summary statistics of the input dataset. This table includes the number of single proteins that did not group with any other in the dataset (singletons). (B) UpSet Diagram showing the number of shared ortholog clusters among the six genomes compared. (C) Total number of proteins and their relative proportions in the top 10 combinations by shared ortholog clusters

A total of 1631 clusters were shared exclusively among all RefSeq genomes which were sequenced with short reads or Sanger sequencing: Phytophthora infestans T304, Phytophthora nicotianae, Phytophthora ramorum, Phytophthora sojae. A set of 1726 proteins from P. infestans T30-4 belonging to these clusters were selected for a tblastn analysis against the assemblies of $P$. betacei
P8084 and P. infestans RC1-10 to test whether or not there was evidence of their presence in those assemblies. For $P$. betacei P8084, tblastn matches were recovered for 1714 proteins $(99.3 \%)$ of which 1683 proteins showed an $\mathrm{H}_{\mathrm{i}}$ value (average between the identity and coverage percentages of the alignment) of $60 \%$ or greater. In $P$. infestans RC1-10, 1706 proteins (98.8\%) showed 
matches against the assembly, of which 1669 proteins showed a $\mathrm{H}_{\mathrm{i}}$ value of $60 \%$ or greater (Supplementary Table 5).

A total of 292 orthologous clusters containing 688 proteins were exclusive to $P$. betacei P8084 (Fig. 2B-C). Of those clusters, only $27(9.24 \%)$ had associated functional terms, mostly with housekeeping functions. Among the GO terms recovered for these clusters, it is possible to find ATP binding (GO:0005524), DNA binding (GO:0003677), sodium ion transport (GO:0006814), L-idonate catabolic process (GO:0046183), carbohydrate: proton symporter activity (GO:0005351), motile cilium (GO:0031514), and protection from non-homologous end joining at telomere (GO:0031848), among others (Supplementary Table 6).

Finally, 124 clusters containing 282 proteins were exclusive to $P$. infestans RC1-10 (Fig. 2B-C), and only 6 clusters ( $4.83 \%$ of the total, namely cluster12486, cluster18538, cluster18539, cluster18587, cluster18612, and cluster18616) had associated functional terms. These terms were related to metabolism and development: protein-lysine $\mathrm{N}$-methyltransferase activity (GO:0016279), zinc ion binding (GO:0008270), long-chain fatty acid-CoA ligase activity (GO:0004467), multicellular organism development (GO:0007275), response to bacterium (GO:0009617), and hydrolase activity (GO:0016787) (Supplementary Table 7).

\section{Phylogeny with single-copy orthologs}

The phylogenetic placement of $P$. betacei P8084 and $P$. infestans RC1-10 within the genus Phytophthora was investigated to provide an evolutionary context for these novel genomes. We selected the 2393 single-copy orthologous clusters shared among the six analyzed assemblies as phylogenetic markers (Supplementary Table 8).
The rooted coalescent-based species tree, inferred from the maximum-likelihood gene trees of each cluster, showed that both $P$. infestans assemblies form a monophyletic group that shares a relatively recent common ancestor with $P$. betacei P8084. The group $P$. infestans $P$. betacei share a common ancestor with $P$. nicotianae (syn. P. parasitica) although the branch length of the former is greater than the latter. Phytophthora sojae appears as the sister group of $P$. nicotianae - P. infestans $P$. betacei, and $P$. ramorum serves as the outgroup relative to all other assemblies (Fig. 3). It is noticeable that the internal branches of the group $P$. nicotianae $-P$. infestans - P. betacei have maximal local posterior probability support.

\section{Whole-genome duplication signals and synteny}

To further explore signatures of evolutionary events leading to the increased genome size and number of genes of $P$. betacei P8084 relative to P. infestans and other species, the distribution of the number of genes per ortholog cluster from the core genome was calculated. It was observed that the assemblies with the largest number of genes: $P$. betacei P8084 (23 457) and $P$. sojae (26 489) (see Fig. 2 A for reference) contain a very large number of genes for certain core orthologous clusters relative to the rest of the compared species (Fig. 4 A). Specifically, P. betacei P8084 had 65 genes in a single core orthologous cluster which is the maximum value for all the genomes analyzed (Supplementary Table 9). In comparison to both assemblies of $P$. infestans, $P$. betacei P8084 had a few more clusters in the range of the $>=50$ paralogs (Fig. $4 \mathrm{~A}$ ). Also, it is very important to notice that $P$. betacei P8084 showed an increase in the number of core orthologous clusters with two genes

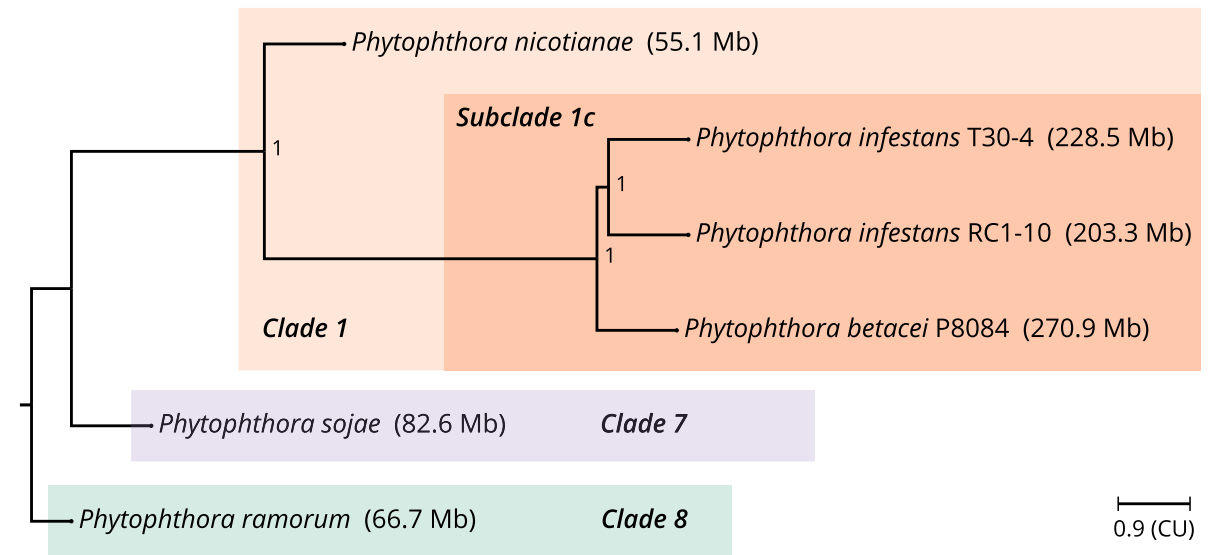

Fig. 3 Phylogenetic placement of Phytophthora betacei P8084 and Phytophthora infestans RC1-10. A coalescent-based species phylogenetic tree was inferred using 2392 maximum-likelihood gene trees derived from single-copy orthologs shared among Phytophthora betacei P8084, P. infestans RC1-10, and other reference assemblies of the genus Phytophthora. The tree was rooted following the topology proposed by Yang et al. [2] and the clade nomenclature was taken from the same study. The scale is in coalescent units (CU), the numbers in the nodes indicate the local posterior probability of the branches, and the assembly size is indicated inside parentheses to the right of the name of the assembly 


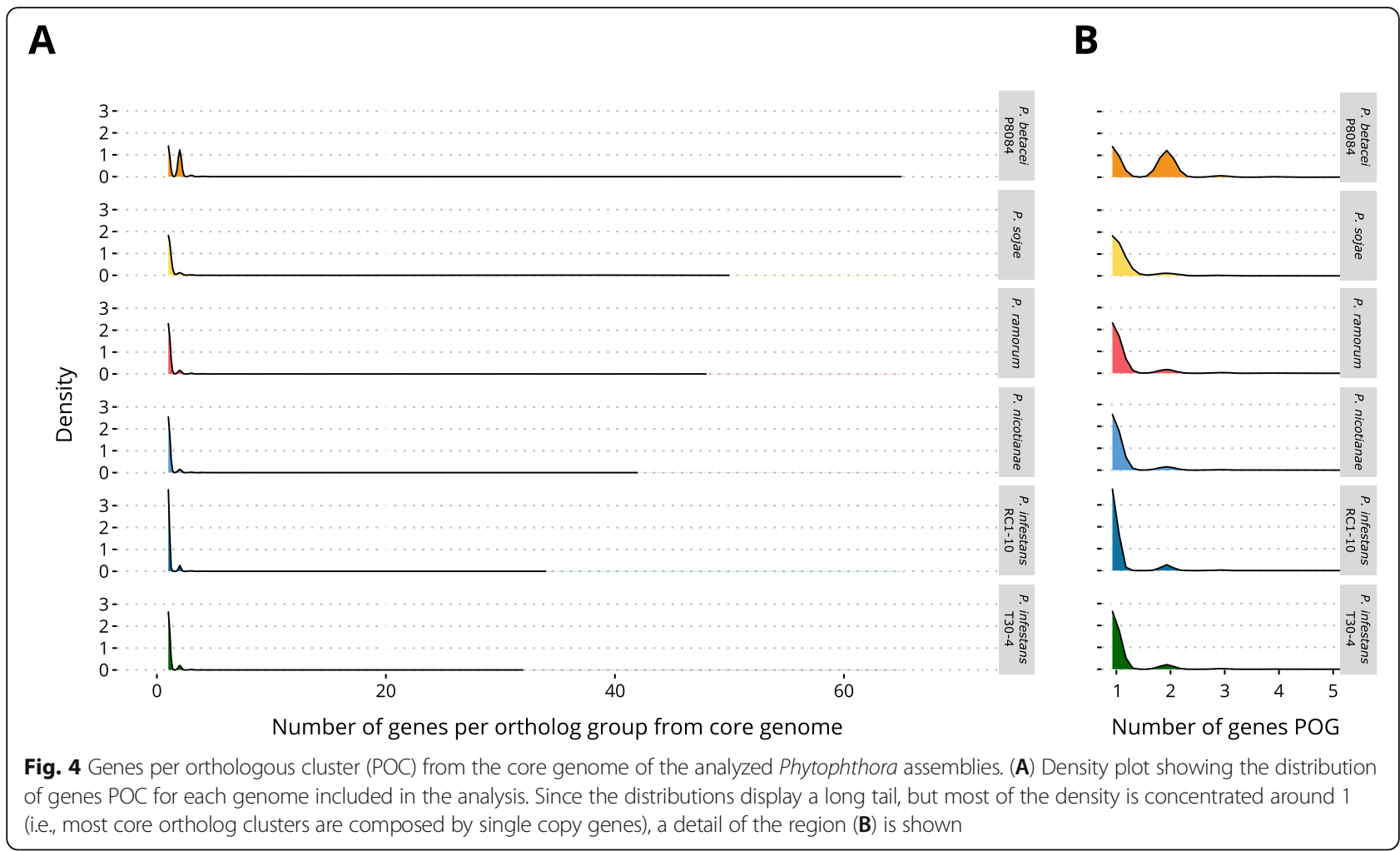

relative to all other genomes analyzed (Fig. 4B), even after haplotig curation.

In order to investigate whether the duplication pattern observed in the core-genome orthologs is a generalized phenomenon, we performed a duplication and collinearity analysis with all the predicted genes for the novel assemblies and compared them to P. infestans T30-4. The genes were classified into the following categories according to their duplication status within the genome: single-copy genes (singletons), dispersed duplicates, proximal duplicates (less than 20 genes apart), tandem duplicates (tandem), and segmental duplications. In $P$. betacei P8084, about half of the genes were contained in duplicated collinear blocks consistent with segmental duplications, and more than a third of the total genes were dispersed duplicates. Around $65 \%$ of the genes in $P$. infestans RC1-10 were dispersed duplicates and almost a quarter of the total genes are singletons. Fewer than $2 \%$ of the genes corresponded to segmental duplications. When comparing these two genomes to $P$. infestans T30-4, it was observed that the proportions of the categories were somewhat conserved among both $P$. infestans assemblies (showing an increase in singletons and tandem repeats in P. infestans T30-4) with differences attributable to sequencing effects but differed greatly from those of P. betacei (Fig. 5 A). A pairwise comparison among P. betacei P8084 and P. infestans RC1-10, revealed that for 300 contigs from the latter assembly there was a median duplication depth $>1$, indicating that for each gene contained in those contigs there is more than one copy in P. betacei P8084. In some cases, complete contigs from $P$. infestans $\mathrm{RC} 1-10$ are contained in more than one contig from $P$. betacei P8084 (Fig. 5B). This further supports the idea that the genome size of $P$. betacei $\mathrm{P} 8084$ can be explained due to large-scale segmental duplication.

Reciprocal pairwise comparisons of aligned CDS between genomes showed there is further evidence of WGD in $P$. betacei P8084 relative to P. infestans. $40 \%$ of the genes from $P$. infestans RC1-10 aligned to 2 collinear anchor blocks from $P$. betacei P8084 genes, compared to only $5 \%$ in the reciprocal comparison (Fig. 5 C). Most of the genes in P. betacei P8084 (70\%) aligned to only one block from $P$. infestans RC1-10 genes. The same pattern was observed in a pairwise comparison among $P$. betacei P8084 and P. infestans T30-4 (data not shown). This evidence shows a pattern of gene duplication (2:1) in P. betacei $\mathrm{P} 8084$ relative to both $P$. infestans assemblies. A comparison between $P$. infestans RC1-10 and P. infestans T30-4 revealed that no whole genome duplication patterns exist between these two assemblies, since the mode of both distributions of aligned genes is 1 block per gene (1:1 pattern). It is also possible to observe that more unmatched genes exist from $P$. infestans RC1-10 when comparing to $P$. infestans T30-4 than the reverse case, possibly reflecting missing regions in the novel assembly (Fig. 5D). 


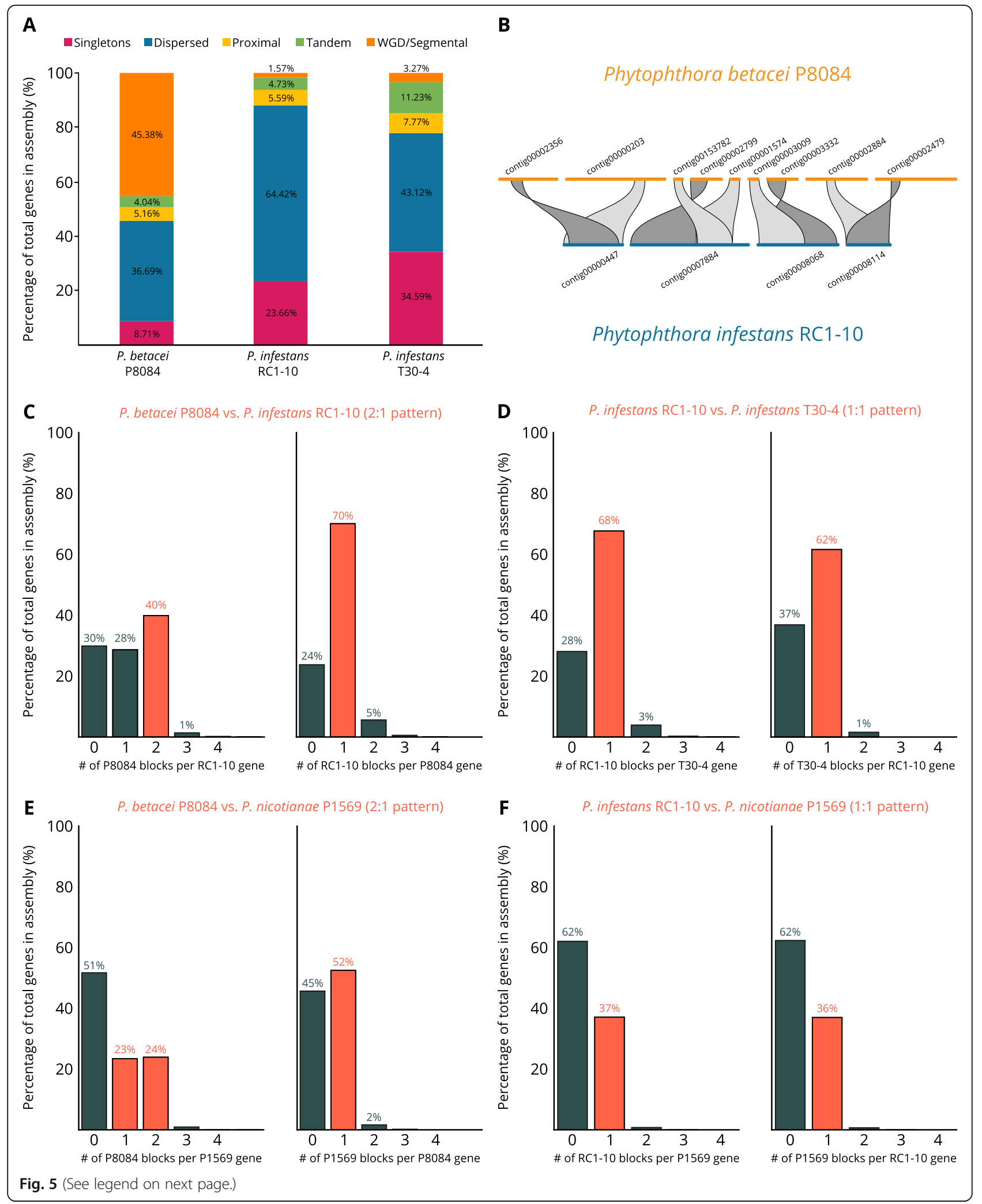


(See figure on previous page.)

Fig. 5 Whole genome duplication in Phytophthora betacei P8084 and comparative synteny analyses. (A) Gene duplication patterns in the assemblies of P. betacei P8084, P. infestans RC1-10, and P. infestans T30-4 shown as the percentage of genes classified in a given duplication type relative to all the predicted genes. (B) Examples of duplicated genomic regions in $P$. betacei P8084 with one single corresponding region in $P$. infestans RC1-10. Histograms showing the distribution of the number of aligned collinear anchor blocks per gene in reciprocal comparisons between: (C) $P$. betacei P8084 and $P$. infestans RC1-10, (D) $P$. infestans RC1-10 and P. infestans T30-4, (E) P. betacei P8084 and P. nicotianae P1569, and (F) P. infestans RC1-10 and P. nicotianae P1569. Bars in orange represent the mode of the distribution for values $>0$ blocks per gene

In Fig. 3 it is very noticeable that a genome-size gap exists between the assemblies of the Subclade 1c and all the others. To assess if there is a genome duplication event that precedes the divergence between $P$. infestans and $P$. betacei, pairwise comparisons of collinear shared blocks were made between our two novel assemblies and P. nicotianae (syn. P. parasitica). Although there is a large fraction of the genes in both assemblies that do not share collinear anchor blocks with $P$. nicotianae and vice versa, it can be observed that there are signals of WGD in $P$. betacei P8084 relative to $P$. nicotianae (Fig. 5E) but a 1:1 pattern is observed between $P$. infestans RC1-10 and P. nicotianae (Fig. 5 F). Additional evidence to support this claim comes from an analysis of rate of synonymous substitutions per synonymous site $(\mathrm{Ks})$ in syntenic collinear gene blocks. In comparisons within the same genome, $P$. betacei P8084 shows a pronounced peak resembling a gaussian bell centered around 0.046 (Fig. 6 A). In contrast, the Ks distributions of both $P$. infestans highly resemble an exponential distribution, with median values lower than $P$. betacei P8084. The difference of collinear gene pairs between the two species is noticeable (Fig. 6B-C). The comparison between P. betacei P8084 - P. infestans RC1-10 and

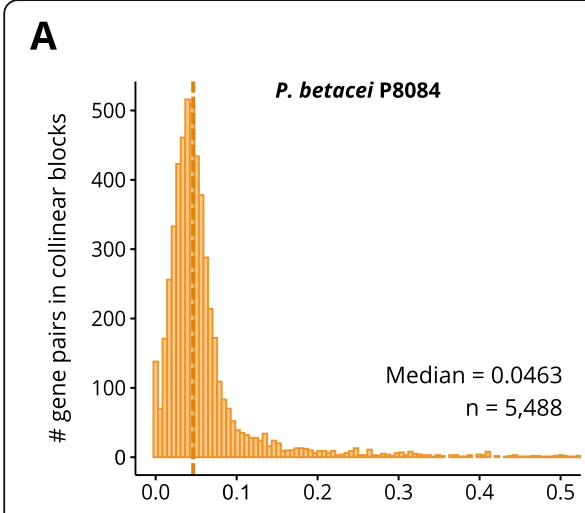

B

E

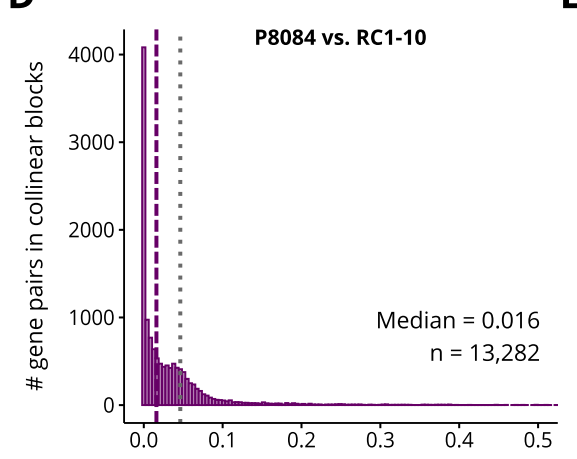

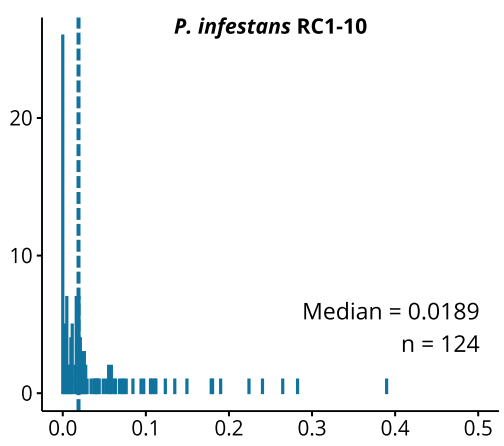

C

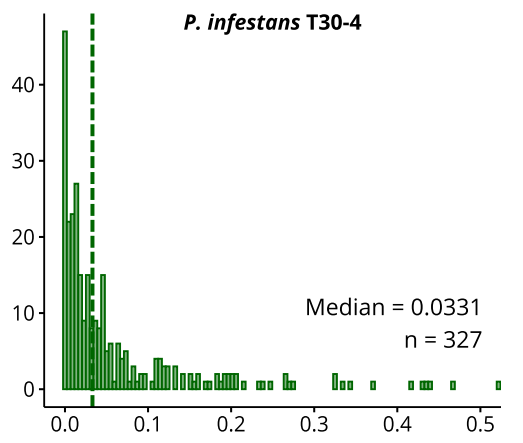

$\mathbf{F}$
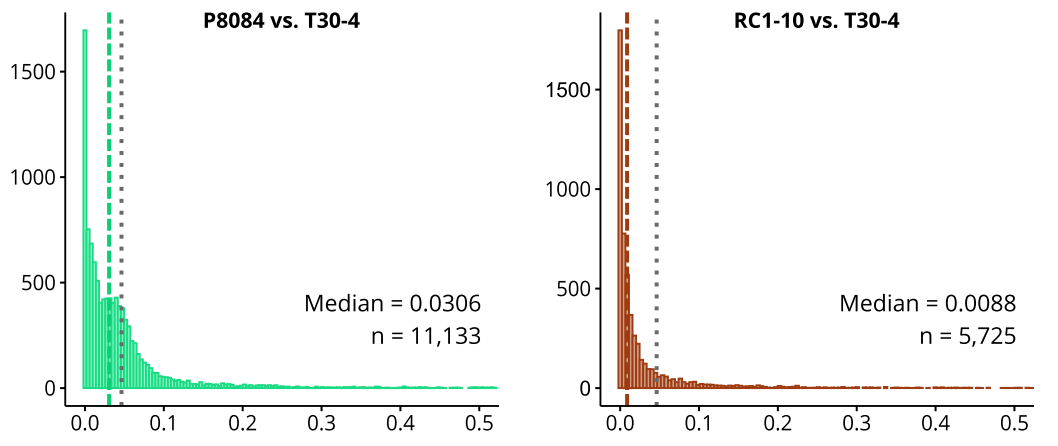

Rate of synonymous substitutions per synonymous site (Ks)

Fig. 6 Synonymous substitutions per synonymous site rate in syntenic blocks from Phytophthora betacei and $P$. infestans. The distribution of Ks was obtained for pairs of genes located in syntenic collinear blocks obtained by MCScanX from alignments within and between genomes. Ks distributions of within-genome comparisons of (A) P. betacei P8084 syntenic blocks, (B) P. infestans RC1-10 syntenic blocks, and (C) P. infestans T30-4 syntenic blocks. Ks distributions of between-genomes comparisons among (D) P. betacei P8084 - P. infestans RC1-10 syntenic blocks, (E) P. betacei P8084 - P. infestans T30-4 syntenic blocks, and (F) P. infestans RC1-10 - P. infestans T30-4 syntenic blocks. The $\mathrm{n}$ represents the number of gene pairs obtained for each comparison. Dashed vertical lines represent the median of the distribution. Dotted vertical lines represent the median of the Ks distribution of P. betacei P8084 blocks represented in $\mathbf{A}$ for comparative purposes 
P. betacei P8084 - P. infestans T30-4 revealed bimodal Ks distributions. In both cases, the tallest peak is centered around $\mathrm{Ks}=0$ and the secondary peak is centered around 0.03 (Fig. 6D-E) which is lower than the median of the distribution of $P$. betacei P8084. The comparison between assemblies of $P$. infestans showed a distribution similar to an exponential one, which is expected for species without WGD (Fig. 6 F). Taken together, all this suggests that $P$. betacei P8084 underwent a lineagespecific WGD process that occurred after the divergence with $P$. infestans.

\section{Transposable elements and short-sequence repeat annotation}

In order to investigate the diversity of transposable and repetitive elements in the genome, the catalog of nonredundant transposable elements (TE) and shortsequence repeats (SSR) of P. betacei P8084 and P. infestans RC1-10 was inferred. For comparison purposes, we also included $P$. infestans T30-4 in the analysis.

Six different orders of retrotransposons (Class I transposons) and five orders of DNA transposons (Class II transposons) could be recovered for all the genomes (Fig. 7). Before filtering potential chimeric elements and potential host genes, Class I transposons comprised $19.6 \%, 43 \%$, and $32.7 \%$ of the total diversity of distinct TEs identified for P. betacei P8084, P. infestans RC1-10, and $P$. infestans T30-4 respectively (Supplementary Table 10). Class II transposons represented $52.4 \%$, $38.9 \%$, and $62.9 \%$ of the total diversity of TEs in P. betacei P8084, P. infestans RC1-10, and P. infestans T30-4, respectively (Supplementary Table 10).

Unclassified repetitive elements (i.e., those that could not be assigned to a particular category) represent $19.3 \%$ and $14.8 \%$ of the total diversity of TEs in P. betacei P8084 and P. infestans RC1-10, respectively. This contrasts with $P$. infestans T30-4 where only $0.5 \%$ of the distinct TEs could not be classified (Fig. 7 A, Supplementary Table 10).

For all TE classes examined in the non-redundant catalog, including uncategorized and potential chimeric elements, $P$. infestans RC1-10 consistently showed a greater number of unique transposon types than P. betacei P8084 and P. infestans T30-4, up to two additional orders of magnitude in certain TE taxonomic orders, despite its smaller genome size (Fig. 7 A). The Long Terminal Repeats (LTR) order of retrotransposons appeared to be the most diverse type of Class I transposons in the three species. For the other main orders of retrotransposons, the number of elements was also higher for $P$. infestans RC110 relative to $P$. betacei $\mathrm{P} 8084$ and P. infestans T30-4. This was particularly noticeable in the case of Large Retrotransposon Derivatives (LARDs) (Fig. 7B). In the case of Short Interspersed Nuclear Elements (SINEs) there was only one type of element for $P$. betacei P8084 and $P$. infestans RC1-10, whereas no SINEs could be found in $P$. infestans T30-4 (Fig. 7B). In Class II transposons, the Terminal Inverted Repeats (TIR) order was the most diverse in all three genomes, although the number of unique TIRs was greater in $P$. infestans RC1-10 relative to the other two assemblies (Fig. 7 C). The three genomes shared a similar degree of diversity for Miniature Invertedrepeat Transposable Elements (MITEs) order. Helitrons were the second most diverse order in P. infestans RC1-10 followed by Cryptons and Mavericks. The second most diverse order of TEs in P. betacei P8084 was MITE followed by Cryptons. A very similar pattern was seen in P. infestans T30-4 (Fig. 7 C). When looking at the number of SSRs across the three genomes, $P$. betacei P8084 was the species with the greatest number of simple repeats relative to both assemblies of $P$. infestans which showed similar values (Fig. 7D). It is interesting to note that a greater diversity of TEs was recovered from $P$. infestans RC1-10 than from $P$. infestans T30-4.

Non-redundant repetitive element catalogs only describe the diversity of the elements found in a genome, but do not explain the contribution of such elements to the genome size of an organism in terms of their copy numbers or their completeness. After matching the TE and SSR catalog to each genome to find the number of copies of each element, it was found that TEs and SSRs comprised $47.31 \%$ of the P. betacei P8084 genome, $68.85 \%$ of the P. infestans RC1-10 genome, and $44.20 \%$ of the $P$. infestans T30-4 genome. These numbers represent $128 \mathrm{Mb}, 141 \mathrm{Mb}$, and $102 \mathrm{Mb}$ of the respective assemblies. In the case of SSRs, a total of 24967 repeats were found for P. betacei P8084, 16224 were found for $P$. infestans RC1-10, and 15563 were found for P. infestans T30-4. A total of 2312 low complexity regions were annotated in P. betacei P8084 compared to 1806 in $P$. infestans RC1-10, and 1609 in $P$. infestans T30-4 (Table 3, Supplementary Table 10).

We calculated the percentage of bases belonging to repetitive elements in $10 \mathrm{~kb}$ non-overlapping windows across the novel assemblies as a first approach to the contribution of such elements to the genome size (Fig. 7E). Most of the windows examined in the three genomes either come entirely from repetitive elements or from regions that do not contain any (the two extremes of the distribution). This can be observed in the histograms of $P$. betacei P8084 and P. infestans RC1-10, as the two largest peaks in both distributions correspond to windows with $0 \%$ and $100 \%$ repetitive content. In contrast, in the histogram of $P$. infestans T30-4 the second largest peak is located near $30 \%$ and it is marginally 


\section{A}

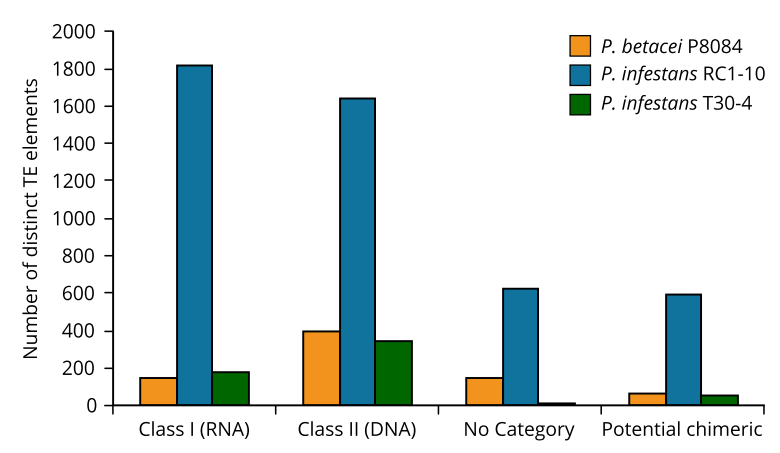

C

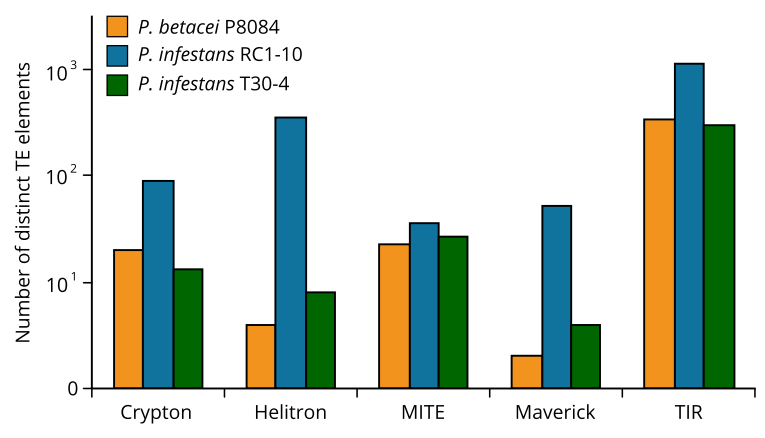

B

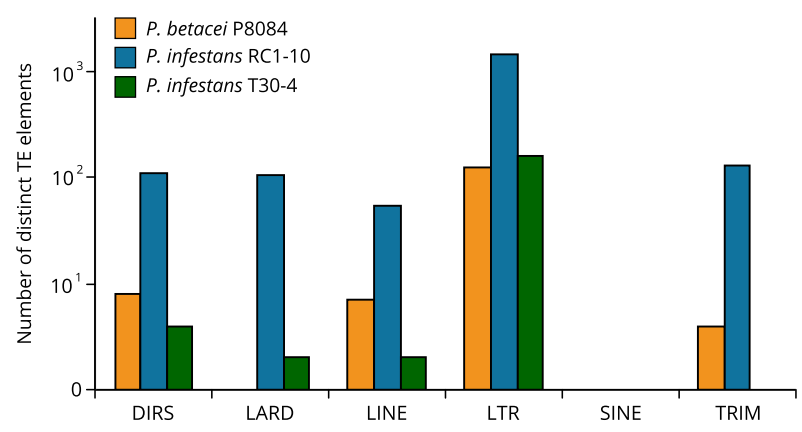

D

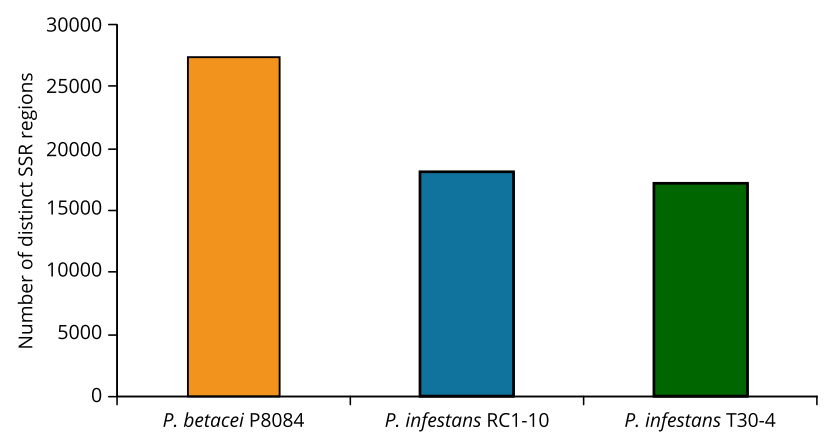

E
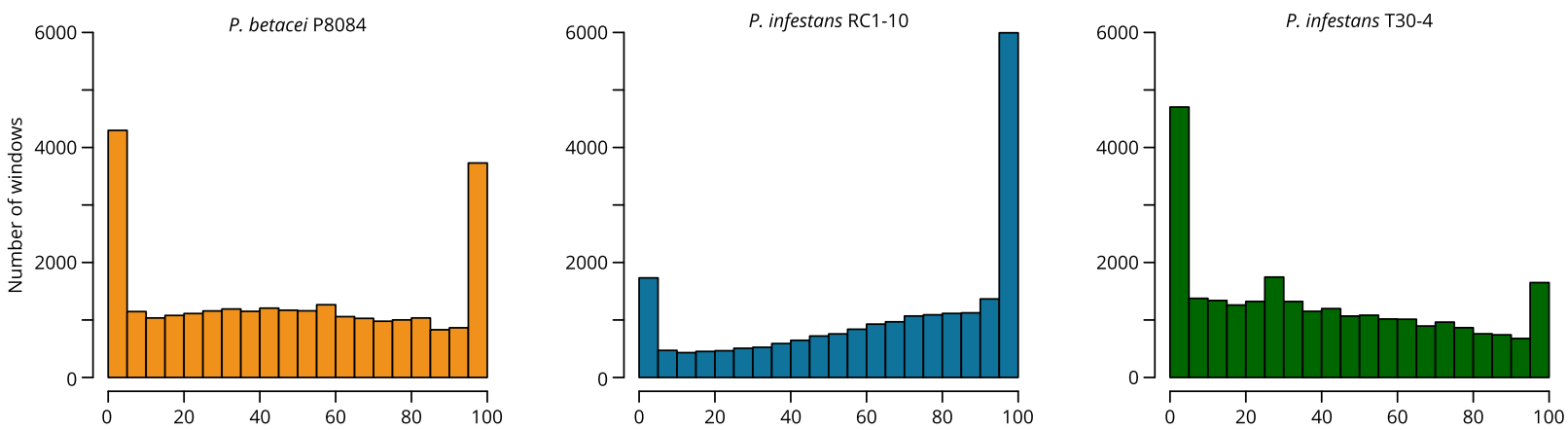

Percentage of TE/SSR content per $10 \mathrm{~Kb}$ window

Fig. 7 Non-redundant catalog of transposable and repetitive elements from Phytophthora betacei P8084 and P. infestans assemblies. (A) Number of distinct transposable elements per class in the catalog. (B) Distinct Class I (RNA transposons or retrotransposons) elements by order. (C) Distinct Class II (DNA transposons) elements by order. (D) Number of distinct short-sequence repeats (SSRs) in the assemblies. (E) Distribution of annotated transposable elements (TEs) and short-sequence repeats (SSRs) in the assemblies of $P$. betacei P8084, $P$. infestans RC1-10, and $P$. infestans T30-4. The data is presented as the percentage of bases annotated as TEs or SSRs per $10 \mathrm{~Kb}$ windows for each genome

larger than the peak at $100 \%$. A higher number of windows that do not come from TEs or SSRs was observed in $P$. infestans T30-4 and P. betacei P8084 relative to $P$. infestans RC1-10. Consistently, a lower number of windows in the first two genomes were part of repetitive elements, being this count notably lower in P. infestans T30-4. Further annotation details can be found in Table 3.

\section{Gene-dense and gene-sparse genomic architecture analysis}

It has been previously reported for other species of genus Phytophthora that their genomes have an architecture characterized by clusters of essential genes for the organism (gene-dense regions, GDRs) separated by highly repetitive regions filled with TEs and SSRs (genesparse regions, GSRs) [8]. In order to investigate the 
Table 3 Annotation statistics of transposable elements (TEs) of Phytophthora betacei P8084 and P. infestans RC1-10 and T30-4 assemblies

\begin{tabular}{|c|c|c|c|}
\hline & $\begin{array}{l}\text { P. betacei } \\
\text { P8084 }\end{array}$ & $\begin{array}{l}\text { P. infestans RC1- } \\
10\end{array}$ & $\begin{array}{l}\text { P. infestans } \\
\text { T30-4 }\end{array}$ \\
\hline Cumulative TE coverage (bp) & 128165366 & 141368243 & 102711821 \\
\hline Percentage of the genome covered by TE (\%) & 47.31 & 68.85 & 44.20 \\
\hline Total number of TE fragments & 75950 & 85415 & 65054 \\
\hline Total number of full-length fragments & $\begin{array}{l}12279 \\
(16.17 \%)\end{array}$ & $11954(14.00 \%)$ & $\begin{array}{l}6314 \\
(9.71 \%)\end{array}$ \\
\hline Total number of TE copies & 63842 & 65956 & 55488 \\
\hline Total number of full-length copies & $\begin{array}{l}13245 \\
(20.75 \%)\end{array}$ & $12961(19.65 \%)$ & $6731(12.13 \%)$ \\
\hline Total number of simple repeats (SSRs) and low complexity regions & 27325 & 18019 & 17172 \\
\hline $\begin{array}{l}\text { Total combined cummulative coverage of simple repeats (SSRs) and low complexity } \\
\text { regions (bp) }\end{array}$ & 1089595 & 744317 & 764909 \\
\hline
\end{tabular}

[This table should appear in the same page than the Results section named Transposable elements and short-sequence repeat annotation]

genomic architecture in $P$. betacei P8084 and P. infestans RC1-10, we measured the 5' and 3' distances between genes, also named flanking intergenic regions (FIRs). Genes that were located inside TEs were excluded from the analysis. Using the FIRs distances, we estimated a genomic distance cut-off such that for a certain gene, if the distance to its closest gene is lower than the cut-off it can be said that it is located within a gene-dense region. On the contrary, if the closest gene is further apart than the cut-off, it is located in a gene-sparse region. In-

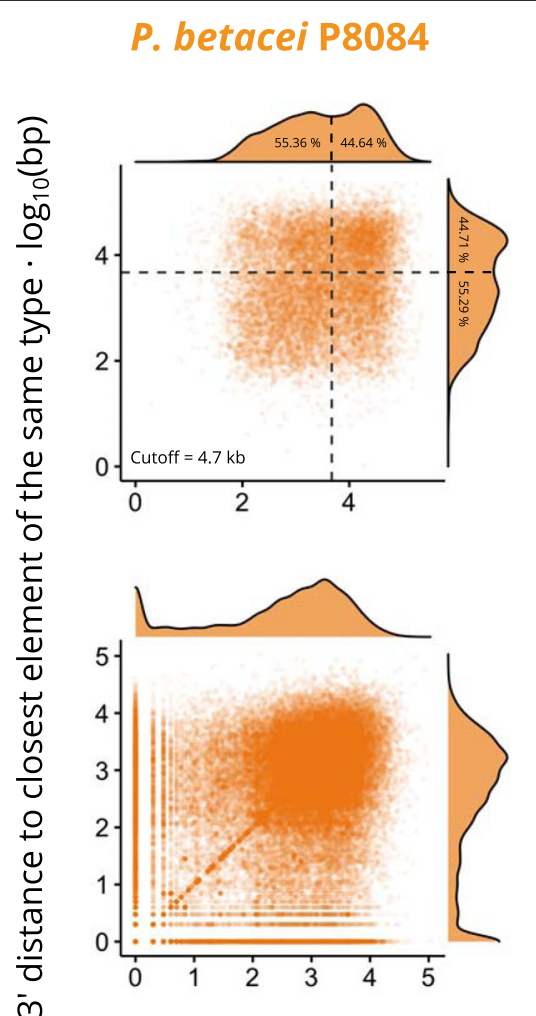

P. infestans RC1-10

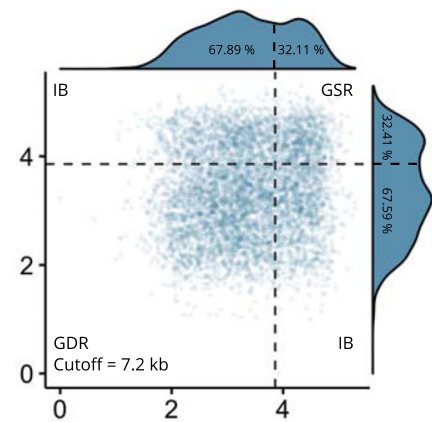

\section{Genes}

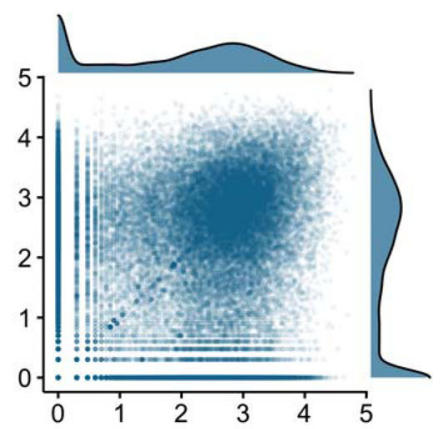

TES

\section{5 ' distance to closest element of the same type $\cdot \log _{10}(\mathrm{bp})$}

Fig. 8 Genome architecture analysis of Phytophthora betacei P8084 and P. infestans RC1-10. Bivariate distributions of the 5' and 3' distances of genes and transposable elements (TEs) to the closest elements of the same type for $P$. betacei P8084 and $P$. infestans RC1-10. Dashed lines represent the estimated flanking intergenic region (FIRs) distance cutoff L used to define gene-dense regions (GDRs), gene-sparse regions (GSRs), and in-between (IB) regions in the assemblies whenever possible. The numbers inside the density plots represent the proportion of the total density of the distribution below and above the value $L$ 
between regions are the border areas where on one side the distance to the next gene is lower than the cut-off, and on the other side it is greater.

Since core orthologs are expected to be enriched in the GDRs, the selection criterion to define the cut-off value $(\mathrm{L})$ was the number of core orthologs located in the putative gene-dense regions of each genome. For $P$. betacei P8084 the calculated cutoff value was $\mathrm{L}=$ $4700 \mathrm{bp}$, and for $P$. infestans $\mathrm{RC} 1-10$ was $\mathrm{L}=7200 \mathrm{bp}$. These were the values that maximized the segregation rate of core orthologs given that the percentage of such genes in GSR or in-between regions did not grow noticeably at higher values of $\mathrm{L}$ (reached saturation). See Supplementary Fig. 3 for more details.

In Fig. 8 a bimodal distribution can be observed for FIRs in both genomes, revealing groupings of genes at distances $\sim 10^{3} \mathrm{bp}$ and another peak at $\sim 10^{5}$ showing more separated genes. In P. betacei P8084 the highest peak is the one located at $\sim 10^{5}$ bp whereas in $P$. infestans RC1-10 the highest one is located around $\sim 10^{3} \mathrm{bp}$. The calculated cutoff value for each genome points to a valley between the two modes.

The 3' and 5' distance measurements were also carried out for the TEs (Fig. 8). In P. betacei P8084 it is possible to observe a high peak of distances around $\sim 10^{3}$ bp and a peak near $\sim 10^{\circ} \mathrm{bp}$. In $P$. infestans RC1-10 a large peak is observed around $\sim 10^{\circ}$ bp with a small peak around $\sim$ $10^{3} \mathrm{bp}$. Peaks around $10^{0} \mathrm{bp}$ are evidence of tightly clustered TEs in both genomes, being much more frequent in P. infestans RC1-10. The peak around $10^{3}$ bp in $P$. betacei P8084 shows that TEs are more spaced between them relative to $P$. infestans RC1-10.

\section{Discussion}

The objective of our study was to sequence and to annotate the related genomes of Phytophthora betacei P8084 and $P$. infestans RC1-10 to understand their pattern of genome divergence and evolution. Phytophthora betacei P8084 is an oomycete plant pathogen with high host preference for Solanum betaceum in natural settings while $P$. infestans RC1-10 was isolated originally from potato and it is believed to have a higher host range. Although these two species are related and belong to the same subclade (clade 1c, which is part of the broader Clade 1, Fig. 3) of the genus Phytophthora, limited gene flow is estimated to occur between them, as evidenced in the original species description [5].

Here, our results showed that $P$. betacei has the largest sequenced genome size of the Phytophthora genus so far with $270 \mathrm{Mb}$. The large genome of $P$. betacei is probably due to an independent species-specific whole genome duplication, whereas $P$. infestans RC1-10 has expanded its genome $(203 \mathrm{Mb})$ relative to the ancestor of the Clade 1 under the activity of transposable elements in the same way described for $P$. infestans T30-4 [12]. However, similar results could be observed from a hybridization between two closely related, yet undescribed, species, but experiments and analysis to discriminate between these possibilities are beyond the scope of the current manuscript.

Several mechanisms contribute to genome size variation in eukaryotes from yeast to more complex genomes like vertebrates. Two of the most important ones are whole genome duplications, due to either autopolyploidy or hybridization, and the accumulation of transposable elements. Whole genome duplications have been extensively studied in plants since it was discovered in the Arabidopsis sequenced genome back in 2000 [20], and it has been reported for oomycetes in a recently published study on P. megakarya and P. palmivora [18]. It is interesting to note that in some cases polyploid genomes are able to return to disomy through a diploidization process such as gene loss, mutation and subfunctionalization, among other mechanisms [21]. These processes have deep consequences on the fate of the paralogous gene copies. WGD is thought to participate in the evolution and adaptation of organisms [22], notably in plants where several studies and methodologies have been developed to detect and understand the relevance of paleo-polyploids in genomic sequences [23].

Our analysis strongly suggests a lineage-specific whole genome duplication in $P$. betacei. We are aware that the large size of the $P$. betacei genome relative to other assemblies in the genus is not in itself a strong criterion to consider the presence of a WGD. However, several lines of evidence suggest that a WGD indeed occurred in $P$. betacei. First, the presence of duplicated genes in the BUSCO analysis, the distribution of genes per core ortholog group across assemblies, and the larger fraction of proteins from $P$. betacei in the core genome led us to consider WGD. This scenario is fully supported by the presence of duplicated blocks in the synteny analysis with $P$. infestans and the Ks curve distribution with a single peak. On the other hand, both Phytophthora infestans assemblies analyzed in this study exhibit genome sizes greater than $200 \mathrm{Mb}$, much larger than those from $P$. nicotianae, $P$. sojae, and $P$. ramorum, but no WGD event was detected in those assemblies. Phytophthora infestans RC1-10 did not show any signal of wholegenome duplication, but extensive dispersed duplications (i.e., non-adjacent duplicated genes) and some short tandem duplicates were observed. A similar duplication pattern was observed for $P$. infestans T30-4, although a smaller proportion of dispersed duplications could be identified in such assembly.

Whole genome duplication signals were previously detected in the Phytophthora genus, suggesting that the process has shaped the evolution of several species or 
even the entire genus. Martens and coworkers predicted the presence of hidden duplications in the ancestor of $P$. infestans, P. sojae and P. ramorum [24] in the form of multiple small blocks of duplicated genes. Such small blocks could suggest extensive rearrangements since the duplication process in the ancestors of the Phytophthora species. However, in a comparative study of small blocks consisting of two directly adjacent genes in Phytophthora, it was postulated that the origin of that kind of duplications is likely to be lineage-specific duplication bursts due to TE activity rather than WGD events [13]. More recently, independent whole genome duplications have been detected in the genomes of $P$. megakarya and P. palmivora, two closely related species in the Phytophthora clade 4, with deep consequences on the expansion of gene families, including RxLR effectors [18].

Similarly to $P$. megakarya and P. palmivora, only one peak was observed in the Ks curve distribution of $P$. betacei suggesting that the cause of the duplication could be an autopolyploidy event rather than allopolyploidy [25]. This WGD event occurred independently from those in P. megakarya and $P$. palmivora in the clade 4 , evidencing that WGD is more frequent than previously thought in the Phytophthora genus. Furthermore, the WGD occurred specifically in $P$. betacei but not in $P$. infestans suggesting a relatively recent event. Although we did not date the WGD in P. betacei, the median value of the Ks distribution in $P$. betacei $(0.046 \mathrm{Ks})$ is similar to the peaks from $P$. megakarya $(0.021 \mathrm{Ks})$ and $P$. palmivora $(0.040 \mathrm{Ks})$. In those two species, duplication was estimated at 0.88 and $1.53 \mathrm{My}$, respectively, long after their estimated date of divergence (16.4 My) and the estimated divergence for the genus (26.6 My) [26].

Although $P$. infestans does not appear to have undergone whole genome duplications, its genome size is large and relatively close to that of $P$. betacei (203 Mb in $P$. infestans $\mathrm{RC} 1-10$ versus $270 \mathrm{Mb}$ ). The origin of this genomic content is found in a high diversity and abundance (in terms of copy number) of transposable elements. These results fit with the current models for genomic expansion in filamentous plant-pathogens that propose the accumulation of TEs and other repetitive sequences as one of the main causes [7]. A large difference in the TE content between P. betacei P8084 and P. infestans RC1-10 is evident, mainly for all categories of retrotransposon families and Helitrons. Long Terminal Repeat retrotransposons transpose via a duplicative mechanism (using an RNA intermediate) that allows them to reach high copy numbers in a genome and are well known to participate in the genome size variation of plants and animals [27]. In rice, the massive amplification of only 3 families of LTR retrotransposons resulted in the doubling of the genome size in a few million years [28]. In conifers, the number of copies of Ty3/Gypsy and Ty1/ Copia LTR retrotransposons accumulated slowly over tens or hundreds of millions of years leading to an increase in the genome sizes of at least 5 species [29].

Differences in the TE content were also observed at the intraspecific level between $P$. infestans RC1-10 and $P$. infestans T30-4. We propose that long-read sequencing technologies allow the access to repetitive elements that previously could not be easily recovered with shortread technologies, most probably due to mis-assembly and assembly collapse. In the latter, the copy number of repetitive transposons in a stretch of DNA is underestimated due to algorithmic limitations of the assemblers [30]. An argument for this comes from the fact that most of the ortholog families found exclusively in the long-read assemblies generated in this study were unclassified genes contained in transposons. Also, an additional hint comes from the presence of hundreds of uncategorized TEs recovered from the two novel assemblies (144 in P. betacei P8084 and 628 in P. infestans RC1-10), in contrast with only 3 found in P. infestans T30-4. Additionally, in our analyses, $P$. infestans T30-4 showed a different genomic transposon content than previously reported. Our TE annotation procedure revealed that only $44.2 \%$ of this genome was composed by TEs, which contradicts the $74 \%$ in the original genome report [12]. The TE annotation strategy used for $P$. infestans T30-4 (RepeatScout) in that report was different to ours, so we attempted to replicate the TE coverage claimed by the original paper using the same tool with no success (data not shown). Taken together, long-read technologies emerge as a new opportunity for research in the field of mobile DNA. We also suggest that there is a need for revision of the TE annotation of the $P$. infestans RefSeq genome.

In the current version of the manuscript, we present a preliminary evaluation of virulence factors, however, in parallel, a thorough analysis was performed on a draft version of our genome by Rojas-Estévez et al. [31]. We thus reference to that manuscript to anyone interested in more detailed analysis of the virulence factors. Nonetheless, we find value in the fact that the number of genes per category of these virulence-related genes was consistent among the $P$. infestans assemblies for the most part. Taking this together with the BUSCO completeness assessments, and the similarities found in the CAZymes and CATAStrophy functional gene profiling, we consider that $P$. infestans RC1-10 assembly is a valuable genomic resource that is semantically comparable to the established $P$. infestans reference genome, and that can be used to delve deeper into the genetics of the EC-1 Andean lineage of the pathogen.

We did not find evidence to support the claim of the GDR/GSR architecture in P. betacei P8084. In this work, we excluded the regions in the genome annotated as TEs and SSRs to obtain a more accurate measurement 
of the FIRs without the bias of including transposon genes. Upon performing the FIR analysis proposed by Raffaelle et al. [8], we found out that the fraction of annotated genes from $P$. betacei P8084 within the putative GSR was not very different from the one in GDR. Also, TEs are more evenly distributed in space in $P$. betacei P8084 than in P. infestans RC1-10, where the GDR/GSR architecture can be observed. We then conclude that there is no evidence to support GDR/GSR architecture in $P$. betacei P8084. As an additional note, the cut-off value $\mathrm{L}$ used to define GSRs that we found for $P$. infestans $\mathrm{RC} 1-10(7.2 \mathrm{~Kb})$ is almost 5 times greater than the one reported in Raffaelle et al. [8]. This is most probably caused, as we specified before, due to the exclusion of TEs from the analysis.

Having an annotated genome allowed us to delve into the phylogenetic relationships of $P$. betacei P8084 by using single-copy orthologs as phylogenetic markers in multispecies coalescent models [32]. In the species description of $P$. betacei by Mideros et al., [5] a SNP phylogeny grouped the candidate $P$. betacei individuals of the species together in a monophyletic clade with $P$. andina as its sister species. Using this approach, $P$. infestans emerged as the sister group of $P$. betacei - $P$. andina. In the same study, a second phylogeny derived from mitochondrial genomes placed $P$. andina and $P$. betacei as a derived lineage of $P$. infestans. Our results provide support for the phylogenetic hypothesis where $P$. betacei emerges as a standalone species. Phylogenomic methods that use orthologs instead of a few selected mitochondrial and nuclear markers still exhibit variability in the placement of major clades within the genus Phytophthora relative to the root (e.g., clades 7 and 8) [33,34]. However, all the species in the Clade 1 show a stable position that is consistent with all phylogenies reviewed in this study and their relationships are well resolved.

Besides allowing phylogenetic inference, the analysis of orthologs (both BUSCO and OrthoVenn analyses) served as a powerful tool for assembly and annotation evaluation. First, the most important hints pointing towards a WGD event in P. betacei P8084 came from the BUSCO duplications and the number of proteins from this assembly in the core genome. Second, finding that one of the largest groups of shared ortholog families was the one composed by orthologs exclusive from shortread assemblies led us to think about the quality of the assembly. In our results, almost all those orthologs could be found as fragmented alignment matches with the nucleotide sequences of both P. betacei P8084 and P. infestans RC1-10. This indicates an opportunity to further improve the quality of these assemblies using complementary sequencing datasets, since these might alleviate the frequent indel errors present in PacBio and long- read sequencing technologies leading to failure in gene prediction by, for example, introducing frameshift errors [35]. Finally, as mentioned before, most of the ortholog families found exclusively in the long-read assemblies generated in this study were unclassified genes contained in transposons which bears testimony of the potential of the long-read technologies to allow the discovery of novel repetitive elements with yet-to-discover functions and implications for the genomes of organisms.

\section{Conclusions}

Two different mechanisms of genome evolution have been identified in two closely related species from the same Phytophthora subclade. Phytophthora betacei P8084 has the largest genome of the genus due to a lineage-specific process of whole genome duplication. The genome size of Phytophthora infestans RC1-10 is the result of the invasion of diverse transposable elements throughout its evolutionary history. These mechanisms are some of the most important drivers of genome architecture, size, and evolution in eukaryotes. Thus, these genomes represent a unique opportunity to study the consequences of such divergent evolutionary paths in the virulence and host range specificity of these pathogens, among many other traits.

We did not find evidence to support the idea that the genome of $P$. betacei P8084 follows the same genedense/gense-sparse architecture proposed for $P$. infestans and other filamentous plant pathogens. One of the questions arising from this fact is whether $P$. betacei represents an exception to the "two-speed genome" hypothesis or if maybe there has not been enough evolutionary time for this genomic dynamic to consolidate. Finally, long-read sequencing is a valuable technology for the study of challenging oomycete genomes, since it allows the access to genomic elements (such as transposable elements) that could not be recovered before due to the limitations of short-read sequencing technologies.

\section{Materials and methods}

\section{Isolates and sequencing}

The biological material used for this study was obtained from the microorganisms collection of the Museum of Natural History of Universidad de los Andes, Colombia, under accession numbers ANDES-F 1172 ( $P$. betacei P8084) and ANDES-F 1833 (P. infestans RC1-10). The usage of the genetic material of Phytophthora betacei P8084 and P. infestans RC1-10 isolates here studied is licensed by the Colombian government to the researchers through the "Contrato de Acceso a Recursos Genéticos y sus productos derivados, $\mathrm{N}^{\circ} 128,23 / 09 / 2016$, MADSSRR". In brief, the origin of the deposited material was for Phytophthora betacei P8084 from a single zoospore isolate obtained from a late blight-affected tree tomato 
(Solanum betaceum) plant in the municipality of Colón, Putumayo, Colombia as described in Mideros et al. [5]. Phytophthora infestans RC1-10 is a single zoospore isolate obtained from an affected potato plant in the municipality of El Rosal, Cundinamarca, Colombia.

The mycelium of each isolate was grown in Plich liquid medium [36] for $15-20$ days in the dark at $20{ }^{\circ} \mathrm{C}$, then harvested, washed, and disrupted in liquid nitrogen. DNA extraction from the disrupted mycelium samples and PacBio Sequel sequencing was carried out by Novogene Corporation (Hong Kong, China) for isolate P8084 and by BGI (Hong Kong, China) for isolate RC1-10. The raw sequencing yield was $\sim 17.5 \mathrm{~Gb}$ for P8084 and $\sim 9.2$ Gb for RC1-10. Additionally, an Illumina PE 100 bp library for P8084 containing $22.7 \mathrm{~Gb}$ sequenced by Mideros et al. [5] was used for assembly polishing and further analyses.

\section{Genome assembly and polishing}

Long reads of both isolates were corrected and assembled using Canu v. 1.6 [37] with the default parameters except for those relative to computing resource usage (30 cores, $128 \mathrm{~GB}$ of RAM). The required genome size value, used in the assembler's correction step, was set to $230 \mathrm{Mb}$ for $P$. infestans RC1-10 given the length of the RefSeq assembly for the species, and to $400 \mathrm{Mb}$ for $P$. betacei $\mathrm{P} 8084$ as an approximate value based on the previous flow cytometry genome size estimations made by Mideros et al. [5].

Two polishing rounds were performed for each assembly. For the first polishing round, the uncorrected sequencing subreads were aligned to the assembly using pbmm2 (https://github.com/PacificBiosciences/pbmm2, v. 0.9.0) wrapper for minimap2 aligner [38]. Variant calling and reference correction were performed using Arrow v. 2.2.2 from the GenomicConsensus package (Pacific Biosciences) with the option for detection of diploid heterozygous variants enabled. Two different approaches were used for the second polishing round: first, Illumina paired reads were aligned to the polished assembly of P8084 with Bowtie2 [39] and variant calling and reference correction was performed using NGSEP v. 3.3.0 [40]; second, the corrected-trimmed subreads obtained from Canu were aligned to the polished assembly of RC1-10, and variant calling and correction was also performed with NGSEP. Genome quality improvement was evaluated by single-copy ortholog analysis with BUSCO v. 3.0.2 [41] with the Stramenopila-Alveolata orthologous gene set, and several assembly statistics were assessed with QUAST v. 4.4 [42].

\section{Haploid representation of the genomes}

Illumina PE data from Phytophthora betacei P8084 was analyzed with GenomeScope v. 2.0 suite using default parameters [43] to obtain an estimation of ploidy and heterozygosity. Assembly curation to obtain a haploid representation of each genome was carried out using the Purge Haplotigs pipeline [44]. Sequencing subreads were aligned to the polished assemblies with pbmm2, the coverage cutoff values to exclude high-coverage misassembled repeats and low-coverage contigs were selected manually following the package documentation. For $P$. betacei P8084 two curation rounds were carried out. In the first round three cutoff values $(60 \%, 70 \%$, and $80 \%)$ were evaluated for their effectivity in haplotig reduction with BUSCO. For the second round the same steps were followed but the alignment cutoffs evaluated were only $70 \%$ and $80 \%$. For $P$. infestans RC1-10 a single purging step was carried out with the same three alignment cutoff values mentioned above, but deactivating the coverage contig filtering option for the $60 \%$ alignment cutoff.

\section{External data sources}

The assemblies and gene annotations of $P$. infestans strain T30-4 (NCBI Assembly Accession: GCA_000142945.1), P. nicotianae (syn. P. parasitica) strain P1569 (NCBI Assembly Accession: GCA_000365505.1), P. sojae strain P6497 (NCBI Assembly Accession: GCA_000149755.2), and P. ramorum strain Pr102 (NCBI Assembly Accession: GCA_000149735.1) were downloaded from the NCBI Assembly repository. Additionally, $P$. infestans T30-4 transcriptome and proteome files were used as evidence for annotation tasks.

\section{Gene prediction and functional annotation}

MAKER 2 (v. 2.31.9) [45] annotation pipeline was used to perform gene prediction and annotation of both $P$. betacei P8084 and P. infestans RC1-10. Within the pipeline, $a b$ initio gene predictions were done using AUGUSTUS v. 3.0.3 [46] on a repeat masked genome, and further refining of gene models was done using $P$. infestans T30-4 transcriptome and proteome derived from RefSeq database (NCBI). Functional assignment of the transcripts was carried out by BLAST searches against UniRef90 database [47] and by protein domain identification using InterProScan v. 5.25-64.0 [48]. The results of these searches were integrated to the predicted gene models using the iprscan2gff3 script included in MAKER 2. Finally, the resulting annotation was further curated by calculating the distribution of the Annotation Edit Distance (AED) values from the GFF file containing the predicted transcripts. A quality filter for the gene models was applied using the MAKER script quality_filtering.pl with an exclusion threshold of AED $>=0.3$. A reannotation of P. infestans T30-4 RefSeq assembly was carried out with the same parameters and input data for comparison purposes.

To refine the annotation quality of proteins relevant to the pathogenesis process in plants, two brief analyses on 
carbohydrate-active enzymes (CAZymes) and virulencerelated genes were performed in the predicted proteomes of the two novel assemblies and the RefSEq. For the CAZymes annotation, a local version of the dbCAN2 server was used to annotate the protein sequences containing domains related to carbohydrate metabolism [49]. This CAZyme analysis was used to classify the organisms into the trophic phenotypes proposed by Hane et al. [19] using the CATAStrophy software (v. 0.0.3) described in such study.

In order to perform a naïve comparison of virulencerelated genes, eight categories were searched in the annotation GFF3 files from $P$. betacei P8084, $P$. infestans RC1-10, and P. infestans T30-4 following the classification proposed by Armitage et al. [50]: Crinkler effectors (CRNs), cutinases, elicitins, necrosis-inducing effectors (NLPs), phytotoxins, protease inhibitors, RxLR effectors, and other virulence-related proteins. The gene search was done with GNU grep command line tool using specific search terms for each category (Supplementary File 1) that included InterPro ontology terms, Pfam terms, and gene names among others.

\section{Analysis of orthologous genes}

The suite OrthoVenn2 [51] was used to obtain clusters of orthologous genes from the novel assemblies $(P$. betacei P8084 and P. infestans RC1-10) and from the selected reference genomes of the genus ( $P$. infestans T304, $P$. sojae, $P$. ramorum, and $P$. nicotianae) using their predicted protein sets as inputs. The visualization of the shared clusters among assemblies was carried out with UpSetR package [52].

To figure out whether the ortholog clusters shared exclusively among $P$. betacei $\mathrm{P} 8084$ and $P$. infestans RC1-10 were derived from transposons, we used BEDTools v. 2.25.0 [53] to subtract the regions annotated as transposons from the MAKER 2 annotation of each genome. Then, we counted the number of genes from the intersection between P. betacei P8084 and P. infestans RC1-10.

A tblastn analysis [54] was run against the assemblies of $P$. betacei P8084 and P. infestans RC1-10 using the set of orthologs of $P$. infestans T30-4 shared exclusively with the other assemblies as input. Individual alignment matches with identity lower than $60 \%$ were filtered out. The coverage percentage of a protein for a certain contig was calculated as the number of uniquely matched positions of the protein, considering all hits in the given contig, divided by its length. The identity percentage of an aligned protein was the average of the identity of all the matches of such protein with the contig. Finally, the harmonic mean between coverage and identity percentages was calculated to obtain a value that we denominated $\mathrm{H}_{\mathrm{i}}$ index. If a protein had matches in more than one contig, only the contig with the best $\mathrm{H}_{\mathrm{i}}$ was selected.

\section{Phylogeny with single-copy orthologs}

A total of 2392 clusters of single copy orthologs shared among all the evaluated assemblies were used to infer a species phylogenetic tree. The proteins of each cluster were aligned with MAFFT v. 7.471 [55] using the GINS-i strategy. Then, the best maximum likelihood tree for each protein cluster was inferred using RAxML v. 8.2.12 [56]. For each tree, the selection of the best amino acid substitution model was done using the -PROTGAMMAAUTO feature of RAxML and a rapid Bootstrap analysis was made with 500 repetitions. After the best 2392 gene trees were obtained, the unrooted coalescent-based species tree was inferred with ASTRAL-III v. 5.7.3 [57]. The tree was rooted following the topology proposed by Yang et al. [2] by assuming a midpoint root that is the last common ancestor between $P$. ramorum (taken as an outgroup for this analysis) and all the other assemblies. Phytophthora sojae and $P$. ramorum were selected on the basis that they belong to the proposed monophyletic clades 7 and 8 of the genus, respectively, that diverged earlier than the proposed clade 1 of which $P$. nicotianae and $P$. infestans that are members [2].

\section{Whole-genome duplication and synteny analyses}

We performed an all-by-all blastp [54] alignment with the proteins coded by the predicted genes within each assembly for P. betacei P8084, P. infestans RC1-10, and $P$. infestans T30-4. The best five non-self hits in each target genome that met an $E$-value threshold of $10^{-5}$ were reported. MCScanX [58] was then used to discover and classify gene duplications in each genome. The compara module of the JCVI utilities (v. 1.0.9 +6.g6732285f) [59] was used to visualize contig-level duplications in $P$. betacei P8084 relative to P. infestans RC1-10, and was also used to obtain histograms of aligned blocks per gene in pairwise comparisons between P. betacei P8084, $P$. infestans RC1-10, P. infestans T30-4, and P. nicotianae (syn. P. parasitica) P1569. This module uses the MCScan pipeline [60] that recovers LAST [61] alignments of the CDSs from predicted genes of each genome to find the best aligned blocks between genomes.

\section{Analysis of synonymous substitutions per synonymous site}

The rate of synonymous substitutions per synonymous site (Ks) was calculated for each one of the duplicated gene pairs found in syntenic collinear blocks identified by MCScanX. This analysis was performed for $P$. betacei P8084, P. infestans RC1-10, and P. infestans T30-4 using the add_ka_and_ks_to_collinearity.pl script included in the MCScanX package. Plots were truncated at $\mathrm{Ks}=2$ for visualization purposes. 
Transposable and repetitive elements annotation

A curated non-redundant catalog of transposable elements (TEs) and repetitive regions in both $P$. betacei P8084 and P. infestans RC1-10 genomes was obtained with REPET TEdenovo v. 2.5 [62] discovery pipeline. Briefly, this pipeline performs structural and sequence similarity searches to find putative TEs, then performs sequence clustering and functional domain annotation to discard false positives and build a final nonredundant catalog. The final step involves taking into account the domain annotation to classify the TEs by type. The annotation of the elements in the genome was done by running REPET TEannot pipeline on the assembly using the non-redundant catalog, filtering all those elements classified as potential host genes and potential chimeric elements. Short-sequence repeats (SSRs) were annotated in the genome by running RepeatMasker [63]. The resulting GFF3 files belonging to each pipeline were merged to build the final repetitive element catalog, and statistics were obtained with TEannot. The same process was performed for $P$. infestans T30-4 for comparison purposes.

\section{Gene-dense and gene-sparse genomic architecture analysis}

The regions with annotated TEs and SSRs were subtracted from the GFF3 files containing the gene annotation for each assembly using BEDTools v. 2.25.0 [53]. This in order to obtain a file that only included TEs and another file containing only genes that were not part of TEs. The distances among TEs and genes, named flanking intergenic regions (FIRs), were calculated from the resulting GFF3 files using a custom script. We then performed a genomic context analysis using the methodology of Raffaele et al. [8] as implemented by RojasEstevez et al. [31]. This analysis aimed to establish a quantitative criterion, the length cutoff (L), to define gene-dense (GDR) and gene-sparse (GSR) regions in both novel assemblies. We first identified single-copy core ortholog clusters using the results obtained in the orthology analysis above. Second, we evaluated different values of $\mathrm{L}$ between $100 \mathrm{bp}$ and $20 \mathrm{kbp}$, and then we computed the percentage of core ortholog genes located in putative GDRs and GSRs relative to the total genes. Third, we calculated the segregation rate defined as the difference between the percentage of all genes in putative GDRs and GSRs, respectively, that are core orthologs. Finally, to select the cutoff $\mathrm{L}$ that best classifies the data, we chose the one that maximizes the segregation rate provided that the number of core ortholog genes classified as either gene-dense or in the boundaries of gene-dense regions (in-between) reached saturation (Supplementary Fig. 3).

\section{Statistics}

All statistical analysis and plots were generated using the stats, base, and ggpubr [64] packages of $\mathrm{R}$ Statistical Computing Language v. 3.5.1 [65] unless otherwise specified.

\section{Supplementary information}

The online version contains supplementary material available at https://doi. org/10.1186/s12864-021-08079-y.

\section{Additional file 1 \\ Additional file 2 \\ Additional file 3 \\ Additional file 4 \\ Additional file $\mathbf{5}$ \\ Additional file 6 \\ Additional file 7 \\ Additional file 8 \\ Additional file 9 \\ Additional file 10 \\ Additional file 11 \\ Additional file 12 \\ Additional file 13 \\ Additional file 14}

\section{Acknowledgements}

The authors thank Prof. Jorge Duitama (Universidad de los Andes, Colombia) and Prof. Diego Riaño-Pachón (CENA, USP, Brazil) for their valuable input and help in genome assembly and phylogenetic inference. The first author also wants to thank Ms. Luisa Matiz Cerón, Ms. Carolina Camelo Valera, Ms. Laura Avellaneda Franco, and for their valuable input and personal support during the writing of the manuscript. Special thanks to the IT Services Department and ExaCore - IT Core facility of the Vice Presidency for Research \& Creation at the Universidad de los Andes for high-performance computing services essential to the research results reported in this work.

\section{Authors' contributions}

D.A.AU participated in the planning and design of the data analysis pipeline, performed most of the computational analysis, participated on the data interpretation and discussion, wrote the first draft of the manuscript; M.C. participated on the data analysis and interpretation, supported the computational analysis; R.G. performed analysis on the transposable element analysis, participated on the data analysis and interpretation. M.C.DM. participated on the planning and design of certain data analyses, participated on the data analysis and interpretation. A.B. participated on the data analysis and interpretation. A.R. participated in the planning and design of the data analysis pipeline, participated on the data analysis and interpretation, performed mentorship and supervision during the project execution, was a major contributor during the writing of the manuscript. S.R. designed and envisioned the project, provide the material and participated on the initial data generation, secured the funding for the project, performed mentorship and supervision during the project execution, participated on the data analysis and interpretation. All authors contributed to the writing or revisions of the manuscript and agree on the final version of it. The author(s) read and approved the final manuscript.

\section{Funding}

This work was funded in part by the Colombian Ministry of Science, Technology, and Innovation (formerly Colciencias) Project No.: 120471250765, Call 712 of 2015; and in part by the grant INV-2018-34-1310 of Facultad de Ciencias at Universidad de los Andes. DAAU was financially supported by the aforementioned Colciencias grant and funds from the Max Planck Tandem Group in Computational Biology and Microbial Ecology. AR was partly funded by the UKRI-BBSRC 'Capacity building for bioinformatics in 
Latin America' (CABANA) grant, on behalf of the Global Challenges Research Fund [BB/P027849/1] which also provided funds for publication, were partly funded by the UKRI-BBSRC 'Capacity building for bioinformatics in Latin America' (CABANA) grant, on behalf of the Global Challenges Research Fund [BB/P027849/1].

\section{Availability of data and materials}

The complete datasets including raw sequencing data, the final assemblies, and the annotation features for the genomes generated in this study have been deposited in the BioProject database of the National Center of Biotechnology Information (NCBI) under the accessions PRJNA608953 for $P$. betacei P8084 and PRJNA517953 for P. infestans RC1-10. All other datasets generated for this study are included in the article and the supplementary material. Further inquiries can be directed to the corresponding authors.

\section{Declarations}

\section{Ethics approval and consent to participate}

Not applicable.

\section{Consent for publication}

Not applicable.

\section{Competing interests}

The authors declare that they have no competing interests.

\section{Author details}

${ }^{1}$ Research Group in Computational Biology and Microbial Ecology, Department of Biological Sciences, Universidad de los Andes, Bogotá, Colombia. ${ }^{2}$ Max Planck Tandem Group in Computational Biology, Universidad de los Andes, Bogotá, Colombia. ${ }^{3}$ Laboratory of Mycology and Plant Pathology (LAMFU), Department of Chemical and Food Engineering, Universidad de Los Andes, Bogotá, Colombia. ${ }^{4}$ Institut de Recherche pour le Développement, CIRAD, Université de Montpellier, 34394 Montpellier, France. ${ }^{5}$ Department of Electronics and Automation, Universidad Autónoma de Manizales, Manizales, Colombia. 'aboratory of Molecular Interactions of Agricultural Microbes (LIMMA), Department of Biological Sciences, Universidad de Los Andes, Bogotá, Colombia. ${ }^{7}$ The Edison Family Center for Genome Sciences and Systems Biology, Washington University School of Medicine, MO 63108 St Louis, USA.

Received: 24 April 2021 Accepted: 27 September 2021 Published online: 05 November 2021

\section{References}

1. Cline ET, Farr DF, Rossman AY. A synopsis of Phytophthora with accurate scientific names, host range, and geographic distribution. Plant Health Prog. 2008;Online. doi:https://doi.org/10.1094/PHP-2008-0318-01-RS.

2. Yang X, Tyler BM, Hong C. An expanded phylogeny for the genus Phytophthora. IMA Fungus. 2017:8:355-84.

3. Fry W. Phytophthora infestans: the plant (and R gene) destroyer. Mol Plant Pathol. 2008:9:385-402.

4. Turner RS. After the famine: Plant pathology, Phytophthora infestans, and the late blight of potatoes, 1845-1960. Hist Stud Phys Biol Sci. 2005;35:341-70.

5. Mideros MF, Turissini DA, Guayazán N, Ibarra-Avila H, Danies G, Cárdenas M, et al. Phytophthora betacei, a new species within Phytophthora clade 1c causing late blight on Solanum betaceum in Colombia. Persoonia - Mol Phylogeny Evol Fungi. 2018. doithttps// doi.org/10.3767/persoonia.2018.41.03

6. Adler NE, Erselius LJ, Chacón MG, Flier WG, Ordoñez ME, Kroon LPNM, et al. Genetic diversity of Phytophthora infestans sensu lato in Ecuador provides new insight into the origin of this important plant pathogen. Phytopathology. 2004;94:154-62.

7. Dong S, Raffaele S, Kamoun S. The two-speed genomes of filamentous pathogens: waltz with plants. Curr Opin Genet Dev. 2015;35:57-65.

8. Raffaele S, Win J, Cano LM, Kamoun S. Analyses of genome architecture and gene expression reveal novel candidate virulence factors in the secretome of Phytophthora infestans. BMC Genomics. 2010;11:637.

9. Evangelisti E, Gogleva A, Hainaux T, Doumane M, Tulin F, Quan C, et al. Time-resolved dual transcriptomics reveal early induced Nicotiana benthamiana root genes and conserved infection-promoting Phytophthora palmivora effectors. BMC Biol. 2017;15:39.
10. Kamoun S. A catalogue of the effector secretome of plant pathogenic oomycetes. Annu Rev Phytopathol. 2006:44:41-60.

11. Kamoun S. Groovy times: filamentous pathogen effectors revealed. Curr Opin Plant Biol. 2007;10:358-65.

12. Haas BJ, Kamoun S, Zody MC, Jiang RHY, Handsaker RE, Cano LM, et al. Genome sequence and analysis of the Irish potato famine pathogen Phytophthora infestans. Nature. 2009;461:393-8.

13. van Hooff JJE, Snel B, Seidl MF. Small homologous blocks in Phytophthora genomes do not point to an ancient whole-genome duplication. Genome Biol Evol. 2014;6:1079-85.

14. Treangen TJ, Salzberg SL. Repetitive DNA and next-generation sequencing: computational challenges and solutions. Nat Rev Genet. 2012;13:36-46.

15. Govers F, Gijzen M. Phytophthora genomics: the plant destroyers' genome decoded. Mol Plant Microbe Interact. 2006;19:1295-301.

16. Nakano K, Shiroma A, Shimoji M, Tamotsu H, Ashimine N, Ohki S, et al. Advantages of genome sequencing by long-read sequencer using SMRT technology in medical area. Hum Cell. 2017;30:149-61.

17. Ali SS, Shao J, Lary DJ, Kronmiller BA, Shen D, Strem MD, et al. Phytophthora megakarya and Phytophthora palmivora, closely related causal agents of Cacao Black Pod Rot, underwent increases in genome sizes and gene numbers by different mechanisms. Genome Biol Evol. 2017;9:536-57.

18. Morales-Cruz A, Ali SS, Minio A, Figueroa-Balderas R, García JF, Kasuga T, et al. Independent whole-genome duplications define the architecture of the genomes of the devastating West African Cacao Black Pod pathogen Phytophthora megakarya and its close relative Phytophthora palmivora. G3 Genes Genomes Genet. 2020;10:2241-55.

19. Hane JK, Paxman J, Jones DAB, Oliver RP, de Wit P. "CATAStrophy," a genome-informed trophic classification of filamentous plant pathogens How many different types of filamentous plant pathogens are there? Front Microbiol. 2020;10. doi:https://doi.org/10.3389/fmicb.2019.03088.

20. Blanc G, Barakat A, Guyot R, Cooke R, Delseny M. Extensive duplication and reshuffling in the Arabidopsis genome. Plant Cell. 2000;12:1093-101.

21. Kuzmin E, VanderSluis B, Ba ANN, Wang W, Koch EN, Usaj M, et al. Exploring whole-genome duplicate gene retention with complex genetic interaction analysis. Science. 2020;368. doi:https://doi.org/10.1126/science.aaz5667.

22. Soltis DE, Albert VA, Leebens-Mack J, Bell CD, Paterson AH, Zheng C, et al. Polyploidy and angiosperm diversification. Am J Bot. 2009;96:336-48.

23. Qiao X, Li Q, Yin H, Qi K, Li L, Wang R, et al. Gene duplication and evolution in recurring polyploidization-diploidization cycles in plants. Genome Biol. 2019;20:38.

24. Martens $C$, Van de Peer $Y$. The hidden duplication past of the plant pathogen Phytophthora and its consequences for infection. BMC Genomics. 2010;11:353.

25. Tiley GP, Barker MS, Burleigh JG. Assessing the performance of Ks plots for detecting ancient whole genome duplications. Genome Biol Evol. 2018;10: 2882-98.

26. Matari $\mathrm{NH}$, Blair JE. A multilocus timescale for oomycete evolution estimated under three distinct molecular clock models. BMC Evol Biol. 2014;14:101.

27. Orozco-Arias S, Isaza G, Guyot R. Retrotransposons in plant genomes: Structure, identification, and classification through bioinformatics and machine learning. Int J Mol Sci. 2019;20:3837.

28. Piegu B, Guyot R, Picault N, Roulin A, Saniyal A, Kim H, et al. Doubling genome size without polyploidization: Dynamics of retrotranspositiondriven genomic expansions in Oryza australiensis, a wild relative of rice. Genome Res. 2006;16:1262-9.

29. Nystedt B, Street NR, Wetterbom A, Zuccolo A, Lin Y-C, Scofield DG, et al. The Norway spruce genome sequence and conifer genome evolution. Nature. 2013:497:579-84.

30. Tørresen OK, Star B, Mier P, Andrade-Navarro MA, Bateman A, Jarnot $P$, et al. Tandem repeats lead to sequence assembly errors and impose multi-level challenges for genome and protein databases. Nucleic Acids Res. 2019;47: 10994-1006.

31. Rojas-Estevez P, Urbina-Gómez DA, Ayala-Usma DA, Guayazan-Palacios N, Mideros MF, Bernal AJ, et al. Effector repertoire of Phytophthora betacei: in search of possible virulence factors responsible for its host specificity. Front Genet. 2020;11. doi:https://doi.org/10.3389/fgene.2020.00579.

32. Li Z, De La Torre AR, Sterck L, Cánovas FM, Avila C, Merino I, et al. Singlecopy genes as molecular markers for phylogenomic studies in seed plants. Genome Biol Evol. 2017;9:1130-47.

33. Sun J, Gao Z, Zhang X, Zou X, Cao L, Wang J. Transcriptome analysis of Phytophthora litchii reveals pathogenicity arsenals and confirms taxonomic status. PLOS ONE. 2017;12:e0178245. 
34. McCarthy CGP, Fitzpatrick DA. Phylogenomic reconstruction of the oomycete phylogeny derived from 37 genomes. mSphere. 2017;2. doi: https://doi.org/10.1128/mSphere.00095-17.

35. Koren S, Phillippy AM, Simpson JT, Loman NJ, Loose M. Reply to 'Errors in long-read assemblies can critically affect protein prediction.' Nat Biotechnol. 2019:37:127-8.

36. van der Lee T, De Witte I, Drenth A, Alfonso C, Govers F. AFLP Linkage Map of the oomycete Phytophthora infestans. Fungal Genet Biol. 1997; 21:278-91.

37. Koren S, Walenz BP, Berlin K, Miller JR, Bergman NH, Phillippy AM. Canu: scalable and accurate long-read assembly via adaptive k-mer weighting and repeat separation. Genome Res. 2017;27:722-36.

38. Li H. Minimap2: pairwise alignment for nucleotide sequences. Bioinformatics. 2018;34:3094-100.

39. Langmead B, Salzberg SL. Fast gapped-read alignment with Bowtie 2. Nat Methods. 2012;9:357-9.

40. Tello D, Gil J, Loaiza CD, Riascos JJ, Cardozo N, Duitama J. NGSEP3: accurate variant calling across species and sequencing protocols. Bioinformatics. 2019;35:4716-23.

41. Waterhouse RM, Seppey M, Simão FA, Manni M, loannidis P, Klioutchnikov $\mathrm{G}$, et al. BUSCO applications from quality assessments to gene prediction and phylogenomics. Mol Biol Evol. 2018;35:543-8.

42. Gurevich A, Saveliev V, Vyahhi N, Tesler G. QUAST: quality assessment tool for genome assemblies. Bioinformatics. 2013;29:1072-5.

43. Vurture GW, Sedlazeck FJ, Nattestad M, Underwood CJ, Fang H, Gurtowski J, et al. GenomeScope: fast reference-free genome profiling from short reads. Bioinformatics. 2017:33:2202-4.

44. Roach MJ, Schmidt SA, Borneman AR. Purge Haplotigs: allelic contig reassignment for third-gen diploid genome assemblies. BMC Bioinformatics. 2018;19:460

45. Holt C, Yandell M. MAKER2: an annotation pipeline and genome-database management tool for second-generation genome projects. BMC Bioinformatics. 2011;12:491

46. Stanke M, Schöffmann O, Morgenstern B, Waack S. Gene prediction in eukaryotes with a generalized hidden Markov model that uses hints from external sources. BMC Bioinformatics. 2006;7:62.

47. Suzek BE, Wang $Y$, Huang $H$, McGarvey PB, Wu CH, the UniProt Consortium. UniRef clusters: a comprehensive and scalable alternative for improving sequence similarity searches. Bioinformatics. 2015;31:926-32.

48. Jones P, Binns D, Chang H-Y, Fraser M, Li W, McAnulla C, et al. InterProScan 5: genome-scale protein function classification. Bioinformatics. 2014;30: $1236-40$.

49. Zhang H, Yohe $T$, Huang L, Entwistle S, Wu P, Yang Z, et al. dbCAN2: a meta server for automated carbohydrate-active enzyme annotation. Nucleic Acids Res. 2018;46:W95-101.

50. Armitage AD, Lysøe E, Nellist CF, Lewis LA, Cano LM, Harrison RJ, et al. Bioinformatic characterisation of the effector repertoire of the strawberry pathogen Phytophthora cactorum. PLoS ONE. 2018;13:e0202305.

51. Xu L, Dong Z, Fang L, Luo Y, Wei Z, Guo H, et al. OrthoVenn2: a web server for whole-genome comparison and annotation of orthologous clusters across multiple species. Nucleic Acids Res. 2019;47:W52-8.

52. Conway JR, Lex A, Gehlenborg N. UpSetR: an R package for the visualization of intersecting sets and their properties. Bioinformatics. 2017;33:2938-40.

53. Quinlan AR, Hall IM. BEDTools: a flexible suite of utilities for comparing genomic features. Bioinformatics. 2010;26:841-2.

54. Altschul SF, Gish W, Miller W, Myers EW, Lipman DJ. Basic local alignment search tool. J Mol Biol. 1990;215:403-10.

55. Katoh K, Standley DM. MAFFT multiple sequence alignment software Version 7: Improvements in performance and usability. Mol Biol Evol. 2013; 30:772-80.

56. Stamatakis A. RAxML version 8: a tool for phylogenetic analysis and postanalysis of large phylogenies. Bioinformatics. 2014;30:1312-3.

57. Zhang C, Rabiee M, Sayyari E, Mirarab S. ASTRAL-III: polynomial time species tree reconstruction from partially resolved gene trees. BMC Bioinformatics. 2018;19:153

58. Wang Y, Tang H, DeBarry JD, Tan X, Li J, Wang X, et al. MCScanX: a toolkit for detection and evolutionary analysis of gene synteny and collinearity. Nucleic Acids Res. 2012:40:e49-e49.

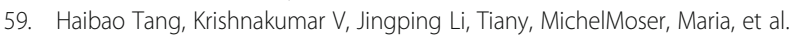
Tanghaibao/Jcvi: Jcvi V0.7.5. Zenodo; 2017. doi:https://doi.org/10.5281/ ZENODO.846919.
60. Tang H, Bowers JE, Wang X, Ming R, Alam M, Paterson AH. Synteny and collinearity in plant genomes. Science. 2008;320:486-8.

61. Kiełbasa SM, Wan R, Sato K, Horton P, Frith MC. Adaptive seeds tame genomic sequence comparison. Genome Res. 2011;21:487-93.

62. Quesneville H, Bergman CM, Andrieu O, Autard D, Nouaud D, Ashburner M, et al. Combined Evidence Annotation of Transposable Elements in Genome Sequences. PLOS Comput Biol. 2005;1:e22.

63. Smit A, Hubley R. RepeatModeler Open-1.0.11. 2017. http://www.repeatma sker.org/.

64. Kassambara A. ggpubr: "ggplot2" Based Publication Ready Plots. 2020. https://CRAN.R-project.org/package=ggpubr.

65. R Core Team. R: A Language and Environment for Statistical Computing. Vienna, Austria: R Foundation for Statistical Computing; 2018. https://www. R-project.org/.

\section{Publisher's Note}

Springer Nature remains neutral with regard to jurisdictional claims in published maps and institutional affiliations.
Ready to submit your research? Choose BMC and benefit from:

- fast, convenient online submission

- thorough peer review by experienced researchers in your field

- rapid publication on acceptance

- support for research data, including large and complex data types

- gold Open Access which fosters wider collaboration and increased citations

- maximum visibility for your research: over $100 \mathrm{M}$ website views per year

At $\mathrm{BMC}$, research is always in progress.

Learn more biomedcentral.com/submissions 